\title{
Functional Impact of the H2A.Z Histone Variant During Meiosis in Saccharomyces cerevisiae
}

\author{
Sara González-Arranz, * Santiago Cavero, * Macarena Morillo-Huesca, ${ }^{\dagger}$ Eloisa Andújar, ${ }^{\ddagger}$ Mónica Pérez- \\ Alegre, ${ }^{*}$ Félix Prado, ${ }^{\dagger}$ and Pedro San-Segundo*,1 \\ *Institute of Functional Biology and Genomics, Consejo Superior de Investigaciones Científicas and University of Salamanca, 37007 \\ Salamanca, Spain and ${ }^{\dagger}$ Department of Genome Biology and ${ }^{\ddagger}$ Genomics Unit, Centro Andaluz de Biología Molecular y Medicina \\ Regenerativa, Consejo Superior de Investigaciones Científicas and University of Seville and University Pablo de Olavide, 41092 \\ Seville, Spain \\ ORCID IDs: 0000-0001-6934-3237 (S.C.); 0000-0001-9805-782X (F.P.); 0000-0002-5616-574X (P.S.-S.)
}

\begin{abstract}
Among the collection of chromatin modifications that influence its function and structure, the substitution of canonical histones by the so-called histone variants is one of the most prominent actions. Since crucial meiotic transactions are modulated by chromatin, here we investigate the functional contribution of the H2A.Z histone variant during both unperturbed meiosis and upon challenging conditions where the meiotic recombination checkpoint is triggered in budding yeast by the absence of the synaptonemal complex component Zip1. We have found that H2A.Z localizes to meiotic chromosomes in an SWR1-dependent manner. Although meiotic recombination is not substantially altered, the $h t z 1$ mutant (lacking H2A.Z) shows inefficient meiotic progression, impaired sporulation, and reduced spore viability. These phenotypes are likely accounted for by the misregulation of meiotic gene expression landscape observed in htz1. In the zip1 mutant, the absence of H2A.Z results in a tighter meiotic arrest imposed by the meiotic recombination checkpoint. We have found that Mec1-dependent Hop1-T318 phosphorylation and the ensuing Mek1 activation are not significantly altered in zip 1 htz1; however, downstream checkpoint targets, such as the meiosis I-promoting factors Ndt80, Cdc5, and Clb1, are drastically downregulated. The study of the checkpoint response in zip 1 htz 1 has also allowed us to reveal the existence of an additional function of the Swe 1 kinase, independent of CDK inhibitory phosphorylation, which is relevant to restrain meiotic cell cycle progression. In summary, our study shows that the H2A.Z histone variant impacts various aspects of meiotic development adding further insight into the relevance of chromatin dynamics for accurate gametogenesis.
\end{abstract}

KEYWORDS Meiosis; meiotic recombination checkpoint; H2A.Z histone variant; gametogenesis; Saccharomyces cerevisiae

EXUAL reproduction relies on a specialized cell division, meiosis, which reduces chromosome ploidy by half and is usually accompanied by cell differentiation processes that culminate in the formation of gametes. The reduction in chromosome complement is achieved by two consecutive rounds of nuclear division preceded by a single round of DNA replication. Premeiotic S-phase is followed by a long prophase I in which, before the first meiotic division, homologous chromosomes (homologs) pair, synapse, and recombine.

Copyright ( 2018 by the Genetics Society of America doi: https://doi.org/10.1534/genetics.118.301110

Manuscript received February 9, 2018; accepted for publication May 29, 2018; published Early Online May 31, 2018.

Supplemental material available at Figshare: https://doi.org/10.25386/genetics. 6376844.

${ }^{1}$ Corresponding author: Instituto de Biología Funcional y Genómica, Consejo Superior de Investigaciones Científicas and University of Salamanca, Calle Zacarías González,

2, 37007 Salamanca, Spain. E-mail: pedross@usal.es
Meiotic recombination is initiated by programmed DNA double-strand breaks (DSBs) generated by the Spo11 protein and a cohort of regulatory factors (Keeney et al. 2014). During the repair of a subset of these meiotic DSBs, crossovers between homologs are formed, which are essential for correct distribution of chromosomes to the meiotic progeny. Alignment of homologous chromosomes (pairing) and the stabilization of these interactions by the synaptonemal complex (SC) (synapsis) influence meiotic recombination outcomes (Hunter 2015). These crucial meiotic events are monitored by the so-called meiotic recombination checkpoint (MRC), an evolutionarily conserved surveillance mechanism that senses defective synapsis and/or recombination and imposes a block or delay in meiotic cell progression providing time to fix the faulty process to prevent aberrant chromosome segregation. The meiotic checkpoint network also operates in unperturbed meiosis to ensure the proper sequential execution of 
events (MacQueen and Hochwagen 2011; Subramanian and Hochwagen 2014).

In this work, we have used the zip1 mutant of the budding yeast Saccharomyces cerevisiae as a genetic tool to activate the MRC. Zip1 is a major structural component of the SC central region and ZIP1 deletion impairs synapsis and crossover (CO) recombination (Dong and Roeder 2000; Borner et al. 2004; Voelkel-Meiman et al. 2015); as a consequence, the zip1 mutant experiences a significant MRC-dependent delay in the prophase to meiosis I transition (Herruzo et al. 2016). The zip1-induced defects are detected by the Mec1-Ddc2 (ATR-ATRIP) complex, resulting in phosphorylation of the Hop1 checkpoint adaptor at several residues, including T318 (Carballo et al. 2008; Refolio et al. 2011; Penedos et al. 2015). The Hop1 protein is a component of the lateral elements of the SC; its abundance, dynamics, and phosphorylation state at chromosome axes in response to checkpoint activation are finely tuned by the AAA+ ATPase Pch2 (Herruzo et al. 2016). Phosphorylated Hop1 recruits the meiosis-specific Mek1 protein to chromosomes facilitating the activation of this Rad53/ Chk2-related kinase containing an FHA domain in two steps: first by Mec1-dependent phosphorylation and subsequently by in trans autophosphorylation of Mek1 dimers on its activation loop (Niu et al. 2005; Ontoso et al. 2013). In turn, active Mek1 stabilizes Hop1-T318 phosphorylation at chromosomes (Chuang et al. 2012). Mek1 promotes interhomolog recombination bias by the direct phosphorylation of the recombination mediator Rad54 at T154 to attenuate its interaction with the strand-exchange Rad51 protein (Niu et al. 2009). Also, the phosphorylation of Hed1 at Thr40 stabilizes this protein stimulating its inhibitory action on Rad51 (Callender et al. 2016). Mek1 also exerts a spatial control on recombination bias by a synapsis-dependent mechanism involving Pch2 (Subramanian et al. 2016). In addition, Mek1 is essential for the meiotic checkpoint response to the accumulation of unrepaired DSBs and to the zip1-induced synapsis and/or recombination defects (Xu et al. 1997; Ontoso et al. 2013; Prugar et al. 2017). The arrest or delay at meiotic prophase I imposed by the MRC is established by two interconnected mechanisms: downregulation of the Ndt80 transcription factor and inhibitory phosphorylation of Cdc28 $8^{\mathrm{CDK} 1}$ (Subramanian and Hochwagen 2014). Ndt80 is a master regulator of yeast meiotic development that activates the transcription of a number of genes involved in meiotic divisions and spore formation (Winter 2012). Among the gene products regulated by Ndt80, the polo-like kinase Cdc5 and the type-B Clb1 cyclin are crucial factors to promote exit from prophase (Tung et al. 2000; Sourirajan and Lichten 2008; Acosta et al. 2011; Argunhan et al. 2017). Inhibition and nuclear exclusion of Ndt80 by the checkpoint prevents the wave of meiotic induction of Clb1 required for entry into meiosis I (Wang et al. 2011). In addition, stabilization of Swe1 by MRC action also maintains $\mathrm{Cdc} 28^{\mathrm{CDK}}$ inhibited by Tyr19 phosphorylation (Leu and Roeder 1999). In sum, the lack of Clb1 induction together with the inhibitory phosphorylation of Cdc28 restrains prophase I exit by keeping in check CDK activity levels.
Most of DNA meiotic transactions occur in the context of highly specialized chromosome and chromatin structures. Chromatin dynamics can be modulated by several processes, including post-translational modification (PTM) of histones and incorporation of histone variants. Among the myriad of histone PTMs described to date, a meiotic function has been ascribed to a number of them (Brachet et al. 2012; Wang et al. 2017). In particular, H3K79 methylation and H4K16 acetylation are involved in the budding yeast MRC (Ontoso et al. 2013; Cavero et al. 2016). Much less is known about the meiotic functional contribution of histone variants, particularly one of the most prominent, H2A.Z, a variant of the canonical histone H2A conserved in evolution from yeast to human.

In vegetative yeast cells, H2A.Z is involved in multiples processes, including transcription regulation (both positively and negatively), maintenance of genome stability, and chromatin silencing (Billon and Côté 2013; Weber and Henikoff 2014). H2A.Z is preferentially found in the vicinity of promoters at nucleosomes flanking a nucleosome-depleted region containing the transcription start site (Raisner et al. 2005). Nevertheless, not all the functions of H2A.Z are necessarily related to transcription; for example, H2A.Z is also deposited at persistent DSBs, promoting their anchorage to the nuclear periphery and stimulating resection (Kalocsay et al. 2009; Adkins et al. 2013; Horigome et al. 2014). The incorporation of H2A.Z to chromatin is carried out by the SWR1 complex, which utilizes the energy of ATP hydrolysis to exchange canonical H2A-H2B by H2A.Z-H2B dimers in particular nucleosomes (Krogan et al. 2003; Kobor et al. 2004; Mizuguchi et al. 2004).

There are few studies addressing the role(s) of H2A.Z during meiosis, although H2A.Z also appears to perform meiotic functions in several model organisms. In Arabidopsis thaliana, H2A.Z is associated to meiotic recombination hotspots and colocalizes with chromosomal foci of the Dmc1 and Rad51 recombinases; moreover, meiocytes from the arp6 mutant (lacking a component of the SWR1 complex) show reduced number of Dmc1, Rad51, and Mlh1 foci, suggesting a role for H2A.Z in the formation and/or processing of meiotic DSBs (Choi et al. 2013). Meiotic gene expression is also altered in the arp6 mutant of A. thaliana (Qin et al. 2014). During mouse spermatogenesis, H2A.Z is first detected at pachytene, but excluded from the sex-body, where it accumulates at later stages. Based on the dynamics of chromosomal distribution during mammalian spermatogenesis, a role for H2A.Z in meiotic sex chromosome inactivation has been proposed (Greaves et al. 2006; Ontoso et al. 2014). Recently, a transcription-independent function of H2A.Z in meiotic DSB generation by modulating chromosomal architecture in the fission yeast Schizosaccharomyces pombe has been reported (Yamada et al. 2018).

In contrast to most organisms where the absence of H2A.Z is not compatible with life, the $h t z 1$ deletion mutant in S. cerevisiae (lacking H2A.Z) is viable, allowing us to directly assess its meiotic functional impact. In most cases, the role of 
H2A.Z in other organisms has been inferred indirectly by analyzing mutants of the SWR1 complex or by cytological observations. In this work, we demonstrate that H2A.Z is important for meiosis in the budding yeast $S$. cerevisiae. We show that the $h t z 1$ mutant displays impaired meiotic progression and sporulation and that spore viability is compromised, although meiotic interhomolog recombination does not appear to be strongly affected. The landscape of gene expression during meiotic prophase is substantially altered in the absence of H2A.Z, likely contributing to at least some of the $h t z 1$ meiotic phenotypes. Finally, we report that H2A.Z also functions during the meiotic checkpoint response induced by the zip 1 mutant affecting the regulators of the prophase to meiosis I transition, such as the Ndt80 transcription factor and the CDK inhibitory kinase Swe1. Our study reveals the existence of novel functional connections between these cell cycle regulators.

\section{Materials and Methods}

\section{Yeast strains}

Yeast strains genotypes are listed in Supplemental Material, Table S1. All of the strains, except the ones used in Figure 2F and Figure S1, are isogenic to the BR1919 background (Rockmill and Roeder 1990). The htz1::hphMX4, swr1::natMX4, swr1:: hphMX4, spo11::natMX4, sum1::natMX4, mer3:hphMX4, swe1:: natMX4, [hta1-htb1]::kanMX6 and [hta2-htb2]::natMX4 gene deletions were made using a PCR-based approach (Longtine et al. 1998; Goldstein and McCusker 1999). The htz1:: URA3 deletion was made using the pTK17 plasmid digested with HindIII-SalI (Santisteban et al. 2000). The zip $1:: L Y S 2$, mek1::kanMX6, ddc2::TRP1, sml1::kanMX6, spo11::ADE2, swe 1::LEU2 and rad51::natMX4 gene deletions were previously described (Leu and Roeder 1999; San-Segundo and Roeder 1999; Refolio et al. 2011; Ontoso et al. 2013; Herruzo et al. 2016). HTZ1-GFP and MIH1-GFP were made by PCR using pKT127 (Sheff and Thorn 2004) and pFA6akanMX6-GFP (Longtine et al. 1998), respectively. The $P_{G A L 1}$-ZIP1-GFP and $P_{G D P 1}$-GAL4(848).ER constructs were obtained from Amy Macqueen (Wesleyan University, CT) (Voelkel-Meiman et al. 2012). Strains carrying Swe1 tagged with three copies of the MYC epitope at the $\mathrm{N}$ terminus and strains carrying ZIP1-GFP have been previously described (Leu and Roeder 1999; White et al. 2004). The kinase-dead swe1-N584A allele was generated using the delitto perfetto approach (Stuckey et al. 2011). Strains carrying the cdc28AF mutation, in which Thr18 and Tyr19 of Cdc28 have been changed to alanine and phenylalanine, respectively, were generated by transformation with the plasmid pR2042 digested with BlpI (Leu and Roeder 1999). The htb1-Y40F mutant strains, in which the Y40 of histone H2B has been mutated to phenylalanine, carry the deletion of the HTA1HTB1 and HTA2-HTB2 genomic loci and a centromeric plasmid (pSS348) expressing HTA1-htb1-Y4OF. These strains were generated as follows. A diploid heterozygous for
[hta1-htb1]::kanMX6 and [hta2-htb2]::natMX4 containing the URA3-based pSS345 plasmid expressing wild-type HTA1-HTB1 was transformed with the TRP1-based pSS347 or pSS348 plasmids expressing wild-type HTA1-HTB1 (as control) or HTA1-htb1-Y4OF, respectively (see plasmid construction below). These diploids were sporulated and UraTrp+ haploid segregants harboring [hta1-htb1]::kanMX6 and [hta2-htb2]::natMX4 genomic deletions and the pSS347 or pSS348 plasmid as the only source for $\mathrm{H} 2 \mathrm{~A}-\mathrm{H} 2 \mathrm{~B}$ or $\mathrm{H} 2 \mathrm{~A}$ $\mathrm{H} 2 \mathrm{BY} 40 \mathrm{~F}$, respectively, were selected. In all cases, gene deletions, mutations, and tagging in haploid strains were made by direct transformation with PCR-amplified cassettes and/or digested plasmids, or by genetic crosses and sporulation (always in an isogenic background) followed by selection of the desired segregants. Diploids were made by mating the corresponding haploid parents and isolation of zygotes by micromanipulation.

\section{Plasmids}

The plasmids used in this work are listed in Table S2. The $2 \mu$ based high-copy pSS248 plasmid contains the meiosis-specific HOP 1 promoter driving the expression of GFP. In-frame cloning of a gene ORF after the GFP in pSS248 leads to overproduction of the GFP-fusion specifically during meiotic prophase. pSS248 was constructed as follows. First, the HOP1 promoter (650 bp) was amplified from genomic DNA and cloned into the BglII-PacI sites of pFA6a-kanMX6-GAL1-GFP (Longtine et al. 1998), replacing the GAL1 promoter by the HOP1 promoter to generate pSS232. Then, the $P_{H O P 1}-G F P$ fragment from pSS232 was amplified by PCR with oligonucleotides containing the appropriate restriction sites and cloned into SpeINotI of pYES2 (Invitrogen, Carlsbad, CA) to replace $P_{G A L 1}$ by $P_{\mathrm{HOP}^{-}}$-GFP generating pSS248. The pSS265 plasmid to overexpress MIH1 during meiosis was constructed by PCR amplification of the MIH1 ORF flanked by NotI-SpeI sites and cloning into NotI-XbaI of pSS248. For meiotic overexpression of BDF1, the ORF flanked by NotI-SphI sites was amplified by PCR and cloned into the same sites of pSS248 to generate pSS354. Plasmid pSS263 was generated by cloning a $2.7-\mathrm{kb}$ NotI-SalI fragment from pSS200 (=p1-1) (Pak and Segall 2002) containing NDT80 plus the promoter and 3'UTR regions into the same sites of the high-copy vector pRS426. The HTA1-HTB1 genomic region containing the genes encoding histones $\mathrm{H} 2 \mathrm{~A}$ and $\mathrm{H} 2 \mathrm{~B}$ expressed from a common divergent promoter including 285 and 540 bp of the flanking 3'UTR sequences was amplified by PCR from genomic DNA and cloned into the BamHI-SalI sites of the centromeric vectors pRS316 and pRS314 to generate plasmids pSS345 and pSS347, respectively. The htb1-Y40F mutation was introduced by site-directed mutagenesis of pSS347 to generate pSS348. All constructs were verified by sequencing. Oligonucleotide sequences are available upon request.

\section{Meiotic time courses, sporulation efficiency, and spore viability}

For meiotic time courses, BR strains were grown in $3.5 \mathrm{ml}$ of $2 \times$ SC medium for $20-24 \mathrm{hr}$ ( $2 \%$ glucose, $0.7 \%$ yeast 
nitrogen base without amino acids, 0.05\% adenine, and complete supplement mixture from Formedium at twice the particular concentration indicated by the manufacturer), then transferred to YPDA (1\% yeast extract, $2 \%$ peptone, $2 \%$ dextrose, $0.02 \%$ adenine) $(2.5 \mathrm{ml})$ and incubated to saturation for additional $8 \mathrm{hr}$. Cells were harvested, washed with $2 \%$ potassium acetate $(\mathrm{KAc})$, resuspended into $2 \% \mathrm{KAc}(10 \mathrm{ml})$, and incubated at $30^{\circ}$ with vigorous shaking to induce meiosis and sporulation. Both YPDA and 2\% KAc were supplemented with $20 \mathrm{mM}$ adenine and $10 \mathrm{mM}$ uracil. The culture volumes were scaled up when needed. To score meiotic nuclear divisions, samples were taken at different time points, fixed in $70 \%$ ethanol, washed in PBS, and stained with $1 \mathrm{mg} / \mathrm{ml}$ DAPI for $15 \mathrm{~min}$. At least 300 cells were counted at each time point. Meiotic time courses were repeated several times. To induce ZIP1-GFP from the $P_{\text {GAL1 }}$ promoter in strains expressing GAL4.ER, $1 \mathrm{mM}$ $\beta$-estradiol (E2257; Sigma, St. Louis, MO; dissolved in ethanol) was added to the cultures. Sporulation efficiency was quantitated by microscopic examination of asci formation after 3 days on sporulation plates. Both mature and immature asci were scored. At least 300 cells were counted for every strain. Spore viability was assessed by tetrad dissection. At least 144 spores were scored for every strain.

\section{Western blotting}

Total cell extracts were prepared by trichloroacetic acid precipitation from 5-ml aliquots of sporulation cultures as previously described (Acosta et al. 2011). Analysis of Mek1 phosphorylation using Phos-tag gels was performed as reported (Ontoso et al. 2013). The antibodies used are listed in Table S3. The ECL or ECL2 reagents (ThermoFisher Scietific) were used for detection. The signal was captured on films and/or with a ChemiDoc XRS system (Bio-Rad, Hercules, CA).

\section{Fluorescence microscopy}

Immunofluorescence of chromosome spreads was performed essentially as described (Rockmill 2009). For analysis of spindle formation by whole-cell immunofluorescence, the following protocol was used. Cells from meiotic cultures $(1.5 \mathrm{ml})$ were fixed with $3.7 \%$ formaldehyde for $45 \mathrm{~min}$, washed twice with solution A (1.2 M sorbitol, $0.05 \mathrm{M} \mathrm{KH}_{2} \mathrm{PO}_{4}$ ), and resuspended into the same solution containing $0.1 \mathrm{mg} / \mathrm{ml} \mathrm{20T}$ Zymolyase, $0.1 \%$ glusulase, and $0.001 \% \beta$-mercaptoethanol. Samples were incubated at $37^{\circ}$ for $20-30 \mathrm{~min}$, while monitoring spheroplast formation. After two washes with ice-cold solution A, cells were resuspended into $50 \mu$ l of this solution. $25 \mu l$ were deposited onto a polylysine-coated eight-well glass slide and left to stand for $30 \mathrm{~min}$. Liquid was carefully aspirated and the slide was submerged into $-20^{\circ}$ methanol for $6 \mathrm{~min}$ and $-20^{\circ}$ acetone for $30 \mathrm{sec}$, using a Coplin jar. The wells were successively rinsed with $1 \%$ BSA in PBS, $1 \%$ BSA $0.1 \%$ NP-40 in PBS (twice), and 1\% BSA in PBS, and incubated overnight with the anti-tubulin antibody in $1 \%$ BSA-PBS at $4^{\circ}$. Wells were then rinsed as described above, incubated with the secondary antibody for $2 \mathrm{hr}$ at room temperature and rinsed again. A drop of Vectashield containing DAPI (H-1200; Vector Laboratories, Burlingame, CA) was deposited and extended with a coverslip sealed with nail polish. The antibodies used are listed in Table S3. Images of spreads and fixed whole cells were captured with a Nikon Eclipse 90i fluorescence microscope controlled with MetaMorph software and equipped with a Hamamatsu Orca-AG CCD camera and a PlanApo VC $100 \times 1.4$ NA objective. Images of fluorescent spores as well as ZIP1-GFP and HTZ1-GFP live cells were captured with an Olympus IX71 fluorescence microscope equipped with a personal DeltaVision system, a CoolSnap HQ2 (Photometrics) camera, and a 100× UPLSAPO 1.4 NA objective. For Zip1-GFP and Htz1GFP, stacks of 10 planes at $0.4-\mu \mathrm{m}$ intervals were captured. Maximum intensity projections of deconvolved images were generated using the SoftWorRx 5.0 software (Applied Precisions). DAPI images were collected using a Leica DMRXA fluorescence microscope equipped with a Hamamatsu OrcaAG CCD camera and a $63 \times 1.4$ NA objective.

\section{Recombination frequency}

To measure genetic distances in a chromosome VIII interval, we used a spore-autonomous fluorescence assay in SK1 strains as previously described (Thacker et al. 2011). Basically, diploid SK1 cells were patched on YEP-glycerol plates, streaked on YPD plates, and single colonies were inoculated into $2 \mathrm{ml}$ of liquid YPD incubated at $30^{\circ}$ for $20 \mathrm{hr}$. Cells were transferred to $10 \mathrm{ml}$ of YPA (1\% yeast extract, $2 \%$ peptone, $2 \%$ $\mathrm{KAc}$ ), incubated at $30^{\circ}$ for $14 \mathrm{hr}$, and sporulated in $10 \mathrm{ml}$ of $2 \%$ KAc containing $0.001 \%$ polypropylene glycol to prevent aggregation. Asci with fluorescent spores were imaged after $48 \mathrm{hr}$ in sporulation. Samples were sonicated for $15 \mathrm{sec}$ before imaging. The "cell counter" plugin of ImageJ (http:// imagej.nih.gov/ij/plugins/cell-counter.html) was used to manually score the tetrads of each type. Genetic distances (centimorgans) were calculated using the Perkins equation: $\mathrm{cM}=$ $(100(6 \mathrm{NPD}+\mathrm{T})) /(2(\mathrm{PD}+\mathrm{NPD}+\mathrm{T}))$, where PD is the number of parental ditypes, NPD is the number of nonparental ditypes, and $\mathrm{T}$ is the number of tetratypes.

\section{Meiotic transcriptome analysis}

Global analysis of gene expression during meiotic prophase was carried essentially as described (Morillo-Huesca et al. 2010). Briefly, gene expression profiles were determined using the "GeneChip Yeast Genome 2.0 Array" of Affymetrix at CABIMER Genomics Unit (Seville, Spain). Total RNA from meiotic prophase cells (15 hr after meiotic induction) was isolated using the RNeasy Midi kit (Qiagen) and its quality was confirmed by Bioanalyzer 2100 (Agilent Technology). Synthesis, labeling, and hybridization of complementary DNA was performed with RNA from three independent cultures of each strain, following Affymetrix protocols (http:// www.affymetrix.com/analysis/index.affx). Probe signal intensities were extracted from the scanned images and analyzed with the GeneChip Operating Software 1.4.0.036 (Affymetrix). The raw data (CEL files) were preprocessed 
and normalized using the robust multichip average method. Fold-change values $(\log 2)$ and their false discovery rateadjusted $P$-values were calculated with Limma (Linear Models for Microarray Analysis) using the affylmGUI interface. All the statistical analysis was performed using $R$ language and the packages freely available from "Bioconductor Project" (http://www.bioconductor.org). Fold-change cutoffs were analyzed at $95 \%$ confidence levels (false discovery rateadjusted $P$-values $<0.05$ ). All data are MIAME (minimal information about a microarray experiment) compliant and the raw data have been deposited at the Gene Expression Omnibus database at the National Center for Biotechnology Information (http://www.ncbi.nlm.nih.gov/geo/) and are accessible through accession number GSE110022. Gene ontology and functional clustering analyses were performed using DAVID tools (Database for Annotation, Visualization and Integrated Discovery) (Huang et al. 2007).

\section{Quantitative RNA analysis}

The amount of messenger RNA (mRNA) was determined by real-time PCR amplification of complementary DNA, generated by reverse transcription and RNaseH treatment (SuperScript II Reverse Transcriptase; Invitrogen) of the RNA samples obtained for the microarray hybridization analysis. Amplification of ACT1 was used to normalize for differences in the amount of input RNA. Similar results were obtained after normalization with NUP84 (data not shown). Primers were designed using the Primer Express software (Applied Biosystems, Foster City, $\mathrm{CA})$ and their sequence is available upon request.

\section{Statistics}

Unless specified, to determine the statistical significance of differences, we used a two-tailed Student's $t$-test. $P$-values were calculated with the GraphPad Prism 5.0 software. The nature of errors bars in graphical representations and the number of biological replicates $(n)$ is indicated in the corresponding figure legend. For analysis of statistical significance in Venn diagrams, we applied a hypergeometric test.

\section{Data availability}

The authors state that all data necessary for confirming the conclusions presented in the manuscript are represented fully within the manuscript and supplemental information. Strains and plasmids are available upon request. Microarray raw data are deposited at the Gene Expression Omnibus repository under accession number GSE110022 (https://www.ncbi.nlm. nih.gov/geo/query/acc. cgi?acc $=$ GSE110022). Supplemental material available at Figshare: https://doi.org/10.25386/ genetics.6376844.

\section{Results}

\section{H2A.Z localizes to meiotic prophase chromosomes in an} SWR1-dependent manner

To investigate the localization of H2A.Z during meiotic prophase we generated a functional version of this histone var- iant tagged with the green fluorescent protein (GFP). Live wild-type cells observed by fluorescence microscopy $15 \mathrm{hr}$ after meiotic induction (peak of prophase in the BR strain background) displayed the H2A.Z-GFP signal along elongated structures likely corresponding with zygotene-pachytene chromosomes. In contrast, the swr 1 mutant showed diffused H2A.Z-GFP throughout the nucleus (Figure 1A). To explore H2A.Z localization in more detail we performed immunofluorescence of meiotic chromosome spreads (Figure 1B). In wild-type pachytene chromosomes, H2A.Z decorated all chromatin, except a particular region of the genome corresponding to the ribosomal DNA region, as demonstrated by the presence of the nucleolar-enriched Pch2 protein (Herruzo et al. 2016) (Figure 1B, arrows). In contrast, pachytene chromosomes of the swr 1 mutant were largely devoid of chromatinassociated H2A.Z (Figure 1B). These observations indicate that, like in vegetative cells (Krogan et al. 2003; Kobor et al. 2004; Mizuguchi et al. 2004), the SWR1 complex is also required for the deposition of H2A.Z into meiotic chromatin. Occasionally, discrete dots of H2A.Z-GFP accumulation could be observed in swr1 nuclei. The nature and possible functional implication of this SWR1-independent localization of H2A.Z will be described elsewhere. Western blot analysis revealed that global levels of H2A.Z remained fairly constant throughout the whole meiotic program in the wild type; however, they were gradually diminishing in the swr1 mutant (Figure 1C), suggesting that chromatin incorporation stabilizes H2A. $\mathrm{Z}$ during meiosis.

\section{Meiotic progression and sporulation are impaired in the htz1 mutant}

To determine whether H2A.Z plays a role in meiotic progression, we followed the kinetics of meiotic divisions by DAPIstaining of nuclei in wild-type and $h t z 1$ strains. Completion of meiotic divisions was less efficient in the $h t z 1$ mutant compared to the wild type (Figure 2A). Likewise, sporulation efficiency and spore viability were also reduced in $h t z 1$, and asci morphology was altered (Figure 2, B-D). These observations imply that H2A.Z function is required for normal meiotic development. The $h t z 1$ mutant showed a random pattern of spore death, with no predominance of four-, two-, and zero-spore-viable tetrads (Figure 2E), suggesting that the reduced spore viability in $h t z 1$ is not resulting, at least exclusively, from meiosis I nondisjunction events. We also examined crossover recombination in a chromosome VIII interval between CEN8 and THR1 using a microscopic fluorescence assay that is independent of spore viability (Thacker et al. 2011). Recombination frequency in this interval, measured as map distance (centimorgans), was not altered in the $h t z 1$ mutant compared to the wild type. As a control, a crossover-defective mer3 mutant was also included in the assay (Figure 2F and Figure S1). To assess whether the inefficient meiotic progression of $h t z 1$ was a consequence of the activation of the MRC, we combined the absence of H2A.Z with that of Spo11 (lacking recombination-initiating meiotic DSBs) and with that of Mek1 (lacking the main checkpoint 


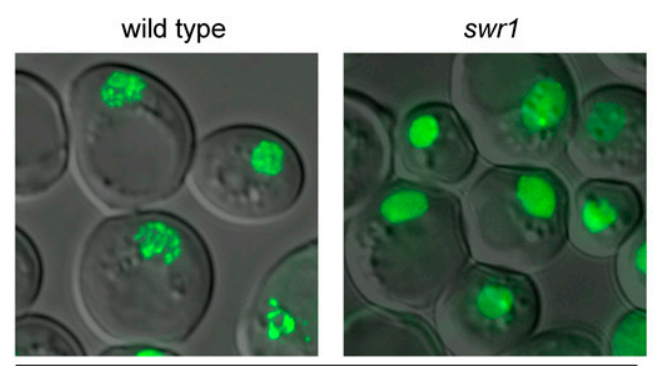

H2A.Z-GFP

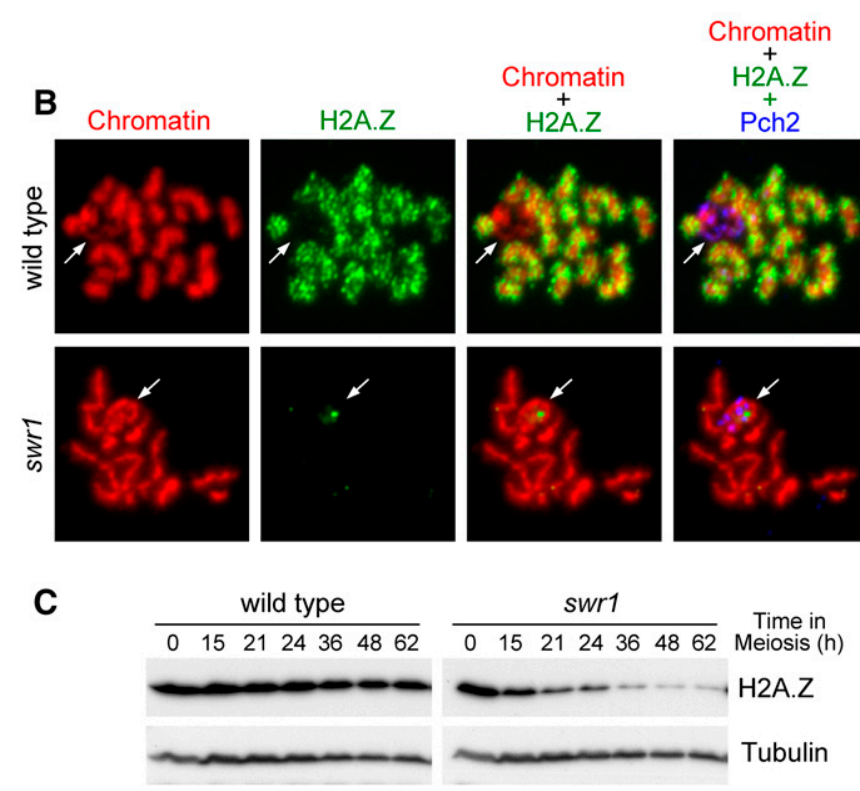

Figure 1 Localization of H2A.Z during meiotic prophase depends on SWR1. (A) Representative images of wild-type and swr1 live cells, at $15 \mathrm{hr}$ after meiotic induction (peak of prophase I), expressing HTZ1GFP. (B) Immunofluorescence of spread meiotic chromosomes from wild type and swr1 stained with DAPI (red) to visualize chromatin, anti-GFP (green) to detect H2A.Z, and anti-Pch2 (blue) to mark the nucleolar region (arrows). (C) Western blot analysis of H2A.Z production during meiosis detected with anti-GFP antibodies. Tubulin was used as a loading control. Strains are DP840 (HTZ1-GFP) and DP841 (swr1 HTZ1-GFP).

effector kinase). The $h t z 1$ delay in meiotic progression was maintained in the $h t z 1$ spol1 and $h t z 1$ mek 1 double mutants (Figure 3, A and B, respectively). Moreover, the dynamics of various indicators of checkpoint activity, such as Hop1-T318 phosphorylation (Herruzo et al. 2016) and Mek1 activation, as assessed both by Mek1 autophosphorylation (Ontoso et al. 2013) and phosphorylation of its H3-T11 target (Cavero et al. 2016; Kniewel et al. 2017), was similar in wild type and $h t z 1$ (Figure 3C). These results indicate that the lower overall efficiency of meiotic divisions in $h t z 1$ does not stem from activation of the MRC, and it is consistent with the observation that $\mathrm{CO}$ meiotic recombination does not appear to be significantly affected in the absence of H2A.Z. To explore the possibility that the absence of H2A.Z affects meiotic entry rather than (or in addition to) meiotic progression, we used ZIP1-GFP as a reporter for early meiotic gene expression and analyzed the percentage of cells showing nu- clear fluorescence in the wild-type and $h t z 1$ strains shortly after meiotic induction. We found that the kinetics of appearance of Zip1-GFP fluorescence was slightly but reproducibly delayed in the $h t z 1$ mutant, although eventually it reached nearly wild-type levels (Figure 3D). This observation likely reflects a delay in the onset of the meiotic program in the absence of H2A.Z and may account, at least in part, for the checkpoint-independent impaired completion of meiotic divisions in the htz 1 mutant.

\section{The SWR1 complex partially impairs meiosis in the absence of H2A.Z}

The SWR1 complex is required to replace H2A-H2B by H2A. $\mathrm{Z}-\mathrm{H} 2 \mathrm{~B}$ dimers at particular nucleosomes. It has been proposed that the SWR1 complex exerts a deleterious effect on chromatin integrity in the $h t z 1$ mutant due to the attempt to replace the canonical histone H2A by H2A.Z in the absence of this histone variant creating nucleosome instability (MorilloHuesca et al. 2010). As a consequence, deletion of SWR1 (encoding the catalytic component of the SWR1 complex) totally or partially suppresses some of the multiple phenotypes of $h t z 1$ in vegetative cells (Morillo-Huesca et al. 2010). Thus, we analyzed the kinetics of meiotic divisions, sporulation efficiency, and spore viability in swr 1 and $h t z 1$ swr $1 \mathrm{mu}-$ tants. We found that meiotic progression was faster (Figure S2A) and that asci formation and spore viability were somewhat improved in $h t z 1$ swr 1 compared to $h t z 1$ (Figure S2, B and $\mathrm{C}$ ), although they did not reach wild-type levels. The swr1 single mutant showed an intermediate phenotype between the wild type and $h t z 1$ mutant in meiotic progression and sporulation efficiency (Figure S2, A and B). These observations imply that some, but not all meiotic phenotypes of $h t z 1$ result from the pathogenic action of SWR1 in the absence of H2A.Z. Moreover, the fact that SWR1 deletion only partially suppresses the meiotic defects of $h t z 1$ also supports a direct impact of H2A.Z chromatin deposition on proper meiotic development.

\section{Meiotic gene expression is altered in the htz1 mutant}

Several studies have demonstrated that mutation of HTZ1 causes transcriptional misregulation during vegetative growth (Billon and Côté 2013). To assess the influence of H2A.Z on general meiotic gene expression, we used whole-genome microarray analysis to compare the transcription profile of wild-type and $h t z 1$ meiotic prophase cells ( $15 \mathrm{hr}$ after meiotic induction). We found 611 genes showing differential expression in $h t z 1$ compared to wild type (1.5-fold cutoff, $P<0.05$ ) (Table S4); of those, 339 genes were upregulated and 272 were downregulated. Among the genes whose expression was increased in the absence of H2A.Z, genes encoding ribosomal proteins were on the top of the list ordered by linear fold change (Table S4). On the top positions of the genes whose expression was significantly downregulated in the $h t z 1$ mutant, we found genes involved in the Mitotic Exit Network (MEN) pathway (BFA1, LTE1) and PP1 phosphatase regulators (GIP4, GAC1) (Table S4). Although there were no 
A

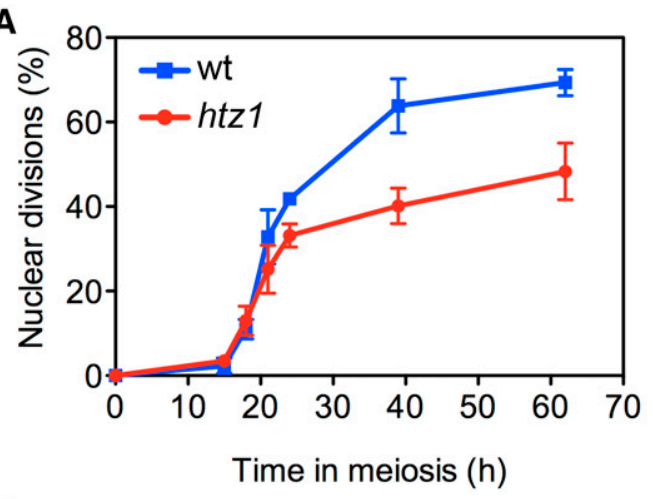

C
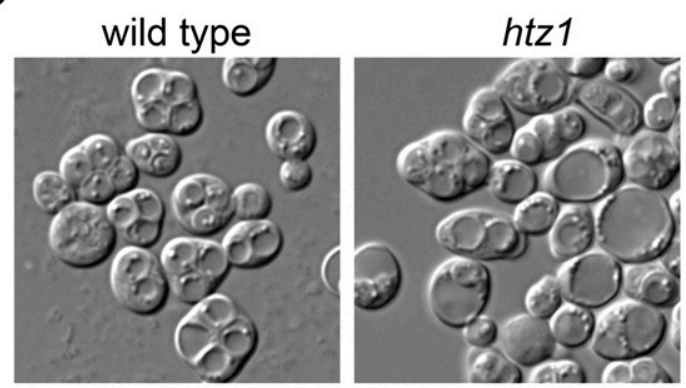

E

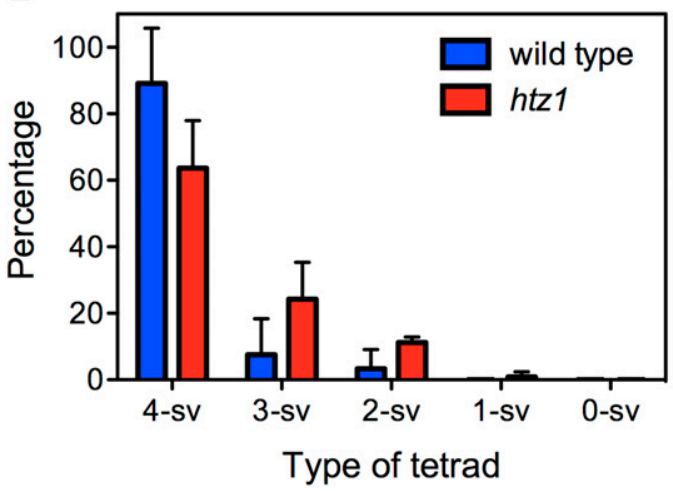

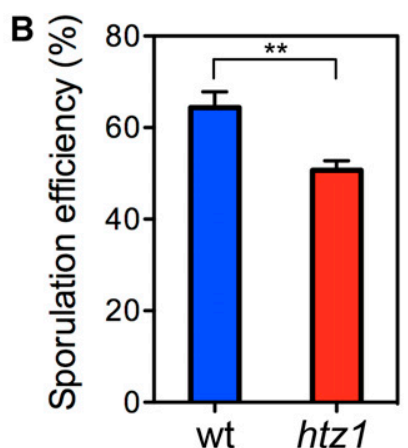

D

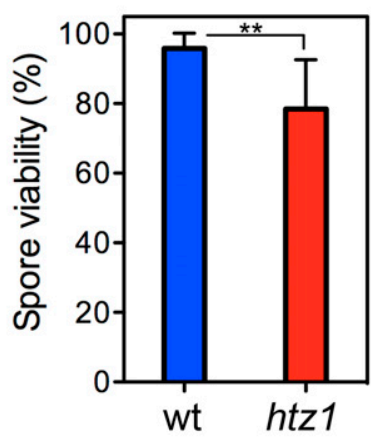

$\mathbf{F}$

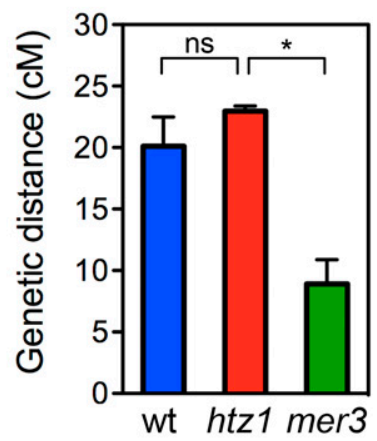

Figure 2 H2A.Z is required for proper meiotic development. (A) Time course analysis of meiotic nuclear divisions; the percentage of cells containing two or more nuclei is represented. Error bars: SD; $n=6$. (B) Sporulation efficiency, determined by microscopic counting, as the percentage of cells forming mature or immature asci after 3 days on sporulation plates. Error bars: SD; $n=3$. (C) Representative DIC images of asci. (D) Spore viability determined by tetrad dissection. At least 288 spores were scored for each strain. Error bars: SD; $n=5$. (E) Distribution of tetrad types. The percentage of tetrads with $4,3,2,1$, and 0 viable spores (4-sv, 3-sv, 2-sv, 1-SV, and 0-sV, respectively) is represented. Error bars: SD; $n=3$. (F) Recombination frequency, expressed as map distance (centimorgans), in a chromosome VIII interval (see Figure S1). Error bars: range; $n=2$. Strains used in A-E are DP421 (wild type) and DP630 (htz1). Strains used in F are DP969 (wild type), DP973 ( $h t z 1)$, and DP974 (mer3). wt, wild type. *, $P<0.05$; **, $P<0.01$ meiosis-specific genes among those whose mRNA levels showed a strong change, it was possible to find some genes with meiotic functions, chromatin, DNA damage response, and cell cycle-related events with a linear fold change $>$ 1.5 (Table 1). The reduced expression of some of these genes in the $h t z 1$ mutant was verified by real-time PCR analysis of the same mRNA samples used in the microarrays (Figure S3A). Moreover, gene ontology and clustering analyses of the genes with decreased expression showed a significant enrichment of functional categories related to both mitotic and meiotic cell cycle regulation (Table S4). On the contrary, genes with increased expression in $h t z 1$ cluster mainly in ribosome biogenesis, translation, and metabolic processes (Table S4). Since genes encoding ribosomal proteins are rapidly repressed upon meiotic induction (Chu et al. 1998), this observation is consistent with the slight delay in meiosis entry of the htz1 mutant (Figure 3D). Interestingly, 133 out of 611 genes $\left(P=5 \times 10^{-5}\right)$ with a differential level of expres- sion between wild type and $h t z 1$ during meiotic prophase identified in this study overlap with those affected by $h t z 1$ (948 genes) in mitotically growing cells (Morillo-Huesca et al. 2010) (Figure S3B).

Thus, these analyses revealed that the meiotic transcriptional landscape is significantly disturbed in $h t z 1$, suggesting that the pleiotropic phenotypes of the $h t z 1$ mutant (aberrant morphology, inefficient meiotic development, low spore viability, etc.) could stem from the more or less subtle alteration of multiple mechanisms.

\section{The zip1 htz1 mutant displays a tight checkpoint- dependent meiotic arrest}

Next, we sought to explore the possible role of H2A.Z during challenged meiosis; that is, under conditions in which meiotic defects trigger the MRC. We used the zip 1 mutant, which is defective in CO recombination and SC formation, to induce the checkpoint. The zip 1 mutant arrests in prophase I for a 


\section{A}

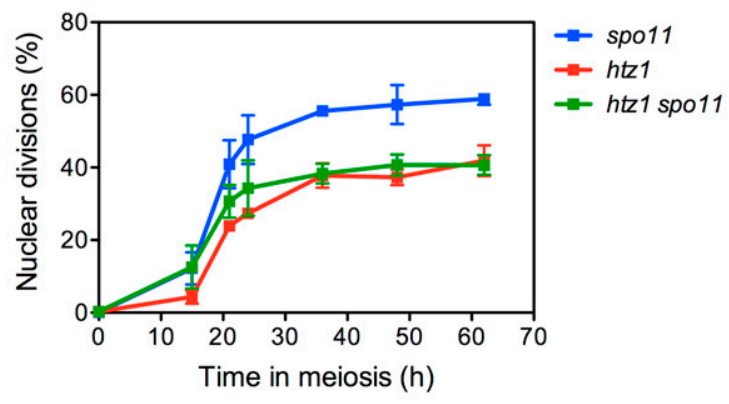

B
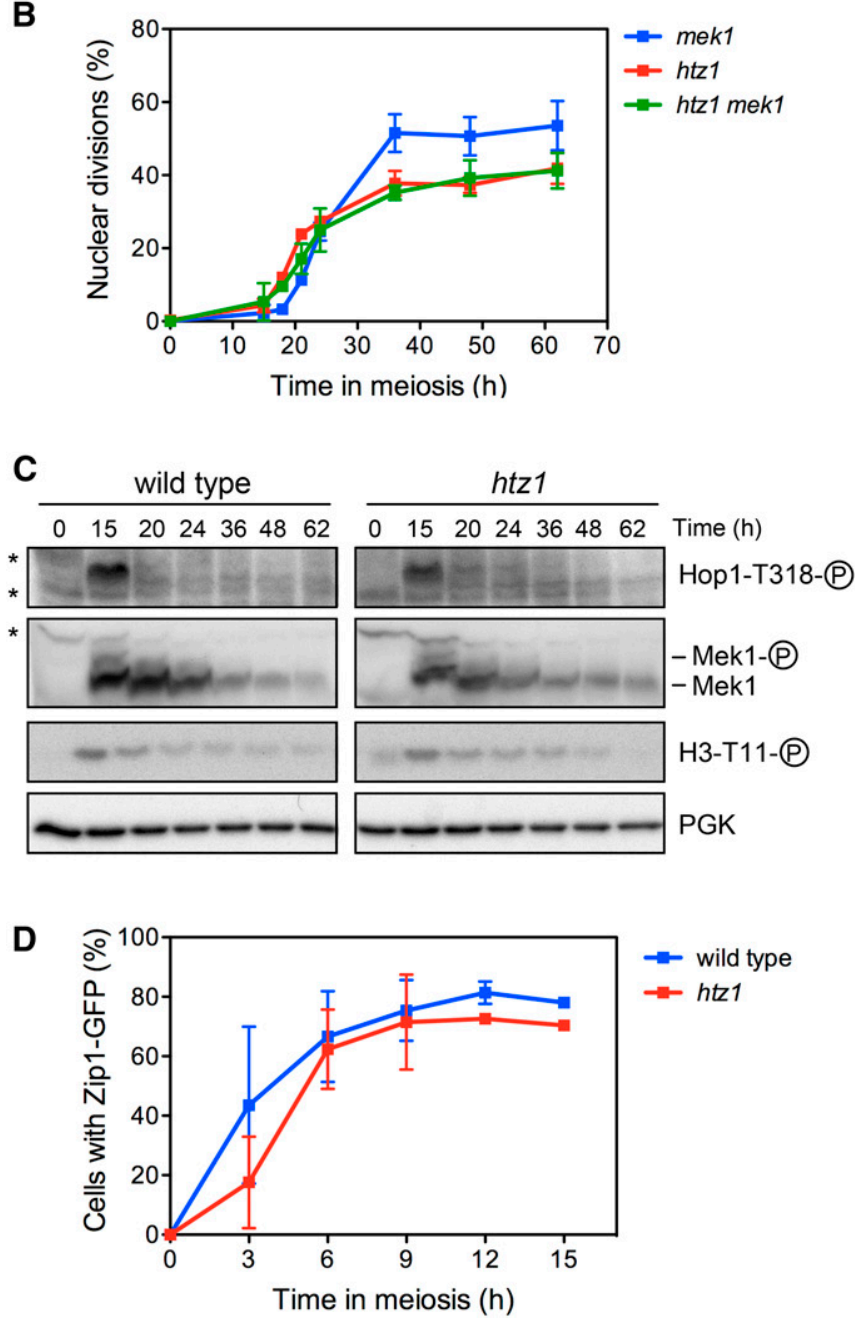

Figure 3 The inefficient meiotic progression of the $h t z 1$ single mutant does not result from activation of the meiotic recombination checkpoint. ( $A$ and $B$ ) Time course analysis of meiotic nuclear divisions; the percentage of cells containing two or more nuclei is represented. Error bars: SD; $n=$ 3. (C) Western blot analysis of Hop1-T318 phosphorylation and Mek1 activity at the indicated time points in meiosis. PGK was used as a loading control. Asterisks mark non-specific bands. Phosphorylated forms are indicated by a circled P. Strains in A-C are DP421 (wild type), DP630 (htz1), DP713 (mek1), DP1523 (spo11), DP1144 (htz1 spo11), and DP1259 (htz1 mek1). (D) Time course analysis of ZIP1-GFP induction. The percentage of cells showing Zip1-GFP nuclear fluorescence during early time points after transfer to sporulation conditions is represented. Strains are DP437 (wild type) and DP838 (htz1). Error bars: SD; $n=3$. long period, but eventually, at late time points, a fraction of the culture completes the meiotic divisions to generate largely inviable spores (Figure 4A) (Ontoso et al. 2013). Strikingly, we found that meiotic progression was completely blocked in the zip $1 \mathrm{htz} 1$ double mutant as most cells remained uninucleated throughout the time course (Figure 4A). This observation suggests that H2A.Z may have a role during prophase I exit because its absence, combined with that of Zip1, provokes a strong meiotic arrest.

Like in the wild type (Figure 1), chromatin incorporation and stability of H2A.Z also depended on SWR1 in the zip1 mutant (Figure S4, A and B). To determine whether the impact of $h t z 1$ on the inability to resume meiotic progression in zip1 was a consequence of the deleterious effect of SWR1 as explained above, we analyzed the kinetics of meiotic divisions in zip1 swr 1 and zip1 htz 1 swr 1 mutants. Interestingly, like zip $1 \mathrm{htz} 1$, the zip 1 swr 1 and zip $1 \mathrm{htz} 1 \mathrm{swr} 1$ mutants also showed a tight meiotic block (Figure S4C). Since the swr1 single mutant is able to complete meiosis, albeit with a small delay compared to the wild type, these results indicate that the strong meiotic arrest of zip $1 \mathrm{htz} 1$, zip $1 \mathrm{swr} 1$, and zip $1 \mathrm{htz} 1$ swr1 stems from the lack of H2A.Z chromatin deposition and does not result from the indirect toxic effect of SWR1 in the absence of H2A.Z.

To ascertain whether the zip $1 \mathrm{htz} 1$ block was caused by the MRC, we generated the zip $1 \mathrm{htz} 1$ spol1 mutant, in which meiotic DSBs are not formed (Keeney et al. 1997), and the zip1 htz1 ddc2 mutant, in which meiotic recombination intermediates are not sensed (Refolio et al. 2011). We found that meiotic divisions and sporulation were largely restored in the zip1 htz 1 spo11 and zip $1 \mathrm{htz} 1 \mathrm{ddc} 2$ mutants (Figure 4B) generating mostly dead spores ( $5.6 \%$ and $1.5 \%$ spore viability for zip1 htz1 spo11 and zip1 htz1 ddc2, respectively; $n=72$ ), thus confirming that the meiotic prophase block in zip $1 \mathrm{htz} 1$ is imposed by the MRC.

\section{The zip1 htz1 mutant does not accumulate additional unrepaired DSBs}

One possible explanation for the more robust meiotic arrest of zip $1 \mathrm{htz} 1$ compared to that of zip 1 is that the absence of H2A. $\mathrm{Z}$ may provoke additional defects that, combined with those resulting from the lack of Zip1, could lead to further hyperactivation of the MRC and, therefore, a tighter prophase I block. To test this possibility, we used immunofluorescence of spread nuclei to analyze the presence of Rad51 foci as an indirect marker for unrepaired DSBs (Joshi et al. 2015) in zip1 and zip1 htz1 mutants. The zip1 htz1 spo11 mutant was also included as a control for the absence of meiotic DSBs. Because of the different kinetics of meiotic progression of the strains analyzed (Figure 4, A and B), only prophase I nuclei, as assessed by the bushy morphology of tubulin staining, were scored (Figure 4C). We found that the zip $1 \mathrm{htz} 1$ double mutant did not display more Rad51 foci than zip1 (Figure 4C), suggesting that the absence of H2A.Z together with that of Zip1 does not generate more unrepaired meiotic DSBs. We also performed immunofluorescence of spread 
Table 1 Subset of genes with decreased meiotic prophase expression in $h t z 1(P<0.05)$

\begin{tabular}{|c|c|}
\hline Gene & $\operatorname{LFC}(>1.5)$ \\
\hline \multicolumn{2}{|l|}{ Cell cycle } \\
\hline BFA1 & 2.393400 \\
\hline LTE1 & 2.277104 \\
\hline GIP4 & 2.268020 \\
\hline$B \cup B 2$ & 1.704342 \\
\hline MCK1 & 1.586536 \\
\hline $\mathrm{MIH1}$ & 1.583281 \\
\hline$C D C 7$ & 1.536321 \\
\hline \multicolumn{2}{|c|}{ Meiotic genes } \\
\hline RME1 & 1.852768 \\
\hline RPD3 & 1.799528 \\
\hline HFM1 & 1.683362 \\
\hline REC8 & 1.666158 \\
\hline MEK1a & 1.599045 \\
\hline MRE11 & 1.597116 \\
\hline SKI8 & 1.570898 \\
\hline IME4 & 1.531359 \\
\hline ZIP2 & 1.511252 \\
\hline SPO22 & 1.504426 \\
\hline HOP1 & 1.499766 \\
\hline \multicolumn{2}{|c|}{ DNA damage response } \\
\hline SRS2 & 1.898810 \\
\hline RAD17 & 1.705357 \\
\hline TOF1 & 1.612276 \\
\hline MEC1 & 1.509767 \\
\hline $\operatorname{IRC6}$ & 1.505446 \\
\hline \multicolumn{2}{|l|}{ Chromatin } \\
\hline SWC3 & 2.864716 \\
\hline SWI3 & 2.070301 \\
\hline$R S C 8$ & 1.850621 \\
\hline SPT20 & 1.631780 \\
\hline HFI1 & 1.629896 \\
\hline CHD1 & 1.514238 \\
\hline
\end{tabular}

LFC, linear fold change.

$a P=0.07$

nuclei using an antibody that recognizes phosphorylated S/T-Q motifs as an additional assay for Mec1/Tel1-dependent DNA damage signaling during meiotic prophase. We found that phospho-S/T-Q foci were significantly increased in a $d m c 1$ mutant, used as a control, that accumulates hyper-resected DSBs (Bishop et al. 1992), but similarly decorated prophase chromosomes of zip1 and zip $1 \mathrm{htz} 1$ (Figure S5). These observations do not favor the possibility that the accumulation of additional DNA damage is responsible for the exacerbated meiotic arrest of zip1 htz1.

\section{Dynamics of upstream checkpoint activation- deactivation is normal in zip1 htz1}

To pinpoint what event in the zip1-induced MRC pathway is affected by H2A.Z, we used a battery of molecular markers to analyze checkpoint status during meiotic time courses of wild type, zip 1, and zip 1 htz 1 strains (Figure 4D). Activation of the Mec1-Ddc2 sensor complex by unrepaired DSBs (and perhaps other types of meiotic defects) is one of the first events in the meiotic checkpoint pathway (Refolio et al. 2011; Subramanian and Hochwagen 2014). Active Mec1 phosphorylates
Hop1 at various sites, including T318 (Carballo et al. 2008; Penedos et al. 2015). In the zip1-induced checkpoint, Hop1T318 phosphorylation is critical to sustain activation of the Mek1 effector kinase (Herruzo et al. 2016), and serves as an excellent readout for Mec1 activity. Since unrepaired DSBs promote Mec1 activation, Hop1 phosphorylation has been also used as an indirect assay for DSB formation (Chen et al. 2015). In the wild type, there was a weak and transient phosphorylation of Hop1-T318 coincident with the peak of prophase I and ongoing recombination. In contrast, Hop1T318 phosphorylation was very robust and sustained in the zip1 mutant (Figure 4D), although at late time points phospho-Hop1-T318 declined coincident with completion of meiotic divisions in a fraction of the culture (Figure 4A). Remarkably, despite the tight meiotic arrest (Figure 4A), the kinetics of Hop1-T318 phosphorylation in the sip $1 \mathrm{htz} 1$ double mutant was similar to that of zip1 (Figure 4D), further supporting that the turnover of meiotic DSBs is not significantly affected by $h t z 1$.

We also monitored the activity of the downstream Mek1 effector kinase using three different readouts: Mek1 autophosphorylation (Ontoso et al. 2013), Hed1 phosphorylation at T40 (Callender et al. 2016), and histone H3 phosphorylation at T11 (Cavero et al. 2016). As shown in Figure 4D, the dynamics of Mek1 activation paralleled that of Hop1-T318 phosphorylation (that is, Mec1 activity) and, again, was similar in both zip 1 and zip $1 \mathrm{htz} 1$, except for a slight persistence of phospho-H3-T11 in zip $1 \mathrm{htz} 1$ at the latest time point.

These results, together with the analysis of Rad51 foci, indicate that the robust meiotic block in zip 1 htz 1 does not arise from the persistence of unrepaired recombination intermediates sustaining permanent upstream checkpoint activation.

\section{H2A.Z is required for reactivation of the cell cycle checkpoint targets}

We next analyzed the downstream targets that are inhibited by the checkpoint to prevent cell cycle progression while recombination and/or synapsis defects persist. In particular, we examined the production of various meiosis I-promoting factors: the Ndt80 transcriptional inductor, the $\mathrm{Clb} 1 \mathrm{cyclin}$, and the Cdc5 polo-like kinase (Acosta et al. 2011). In addition, we also monitored the levels of the Swe1 kinase and its activity: the inhibitory phosphorylation of Cdc28 (CDK) at tyrosine 19 (Leu and Roeder 1999). In the wild type, after the recombination process is completed and the transient activation of Mek1 disappears, the program for meiosis I entry is turned on with the production of Ndt80, Clb1, and Cdc5, as well as the reduction of the inhibitory phosphorylation at Y19 of Cdc28 (Figure 4D). In the zip1 mutant, the induction of Ndt80, Clb1, and Cdc5 were significantly delayed and high levels of the Swe1 kinase promoting Cdc28-Y19 phosphorylation persisted for as long as Mek1 was active. However, as Mek1 activation eventually declined, Ndt80 and Cdc5 were induced, and Swe1 and phosphoCdc28-Y19 diminished, thus sustaining entry into meiosis I of at least a fraction of the cells (Figure 4, A and D). In 
A

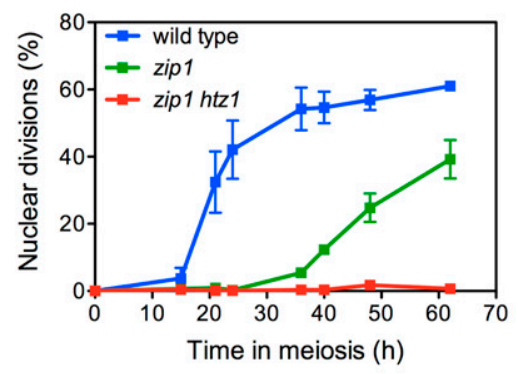

B

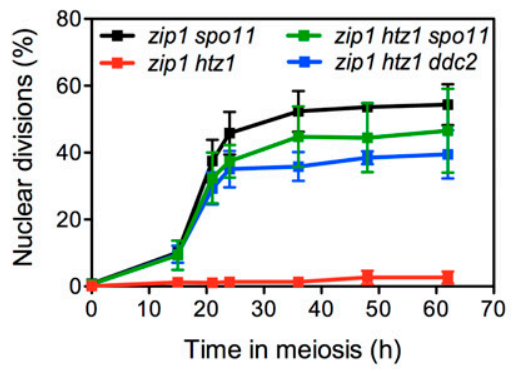

C
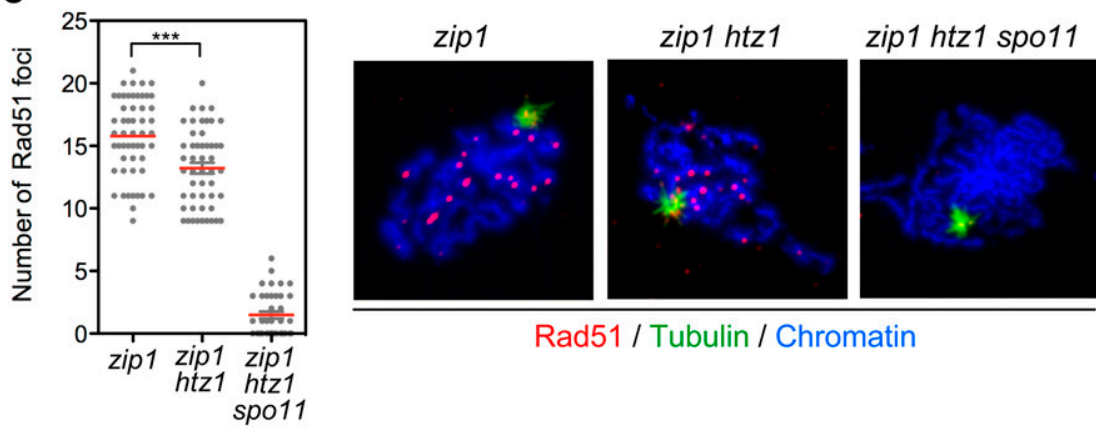

Rad51 / Tubulin / Chromatin

D

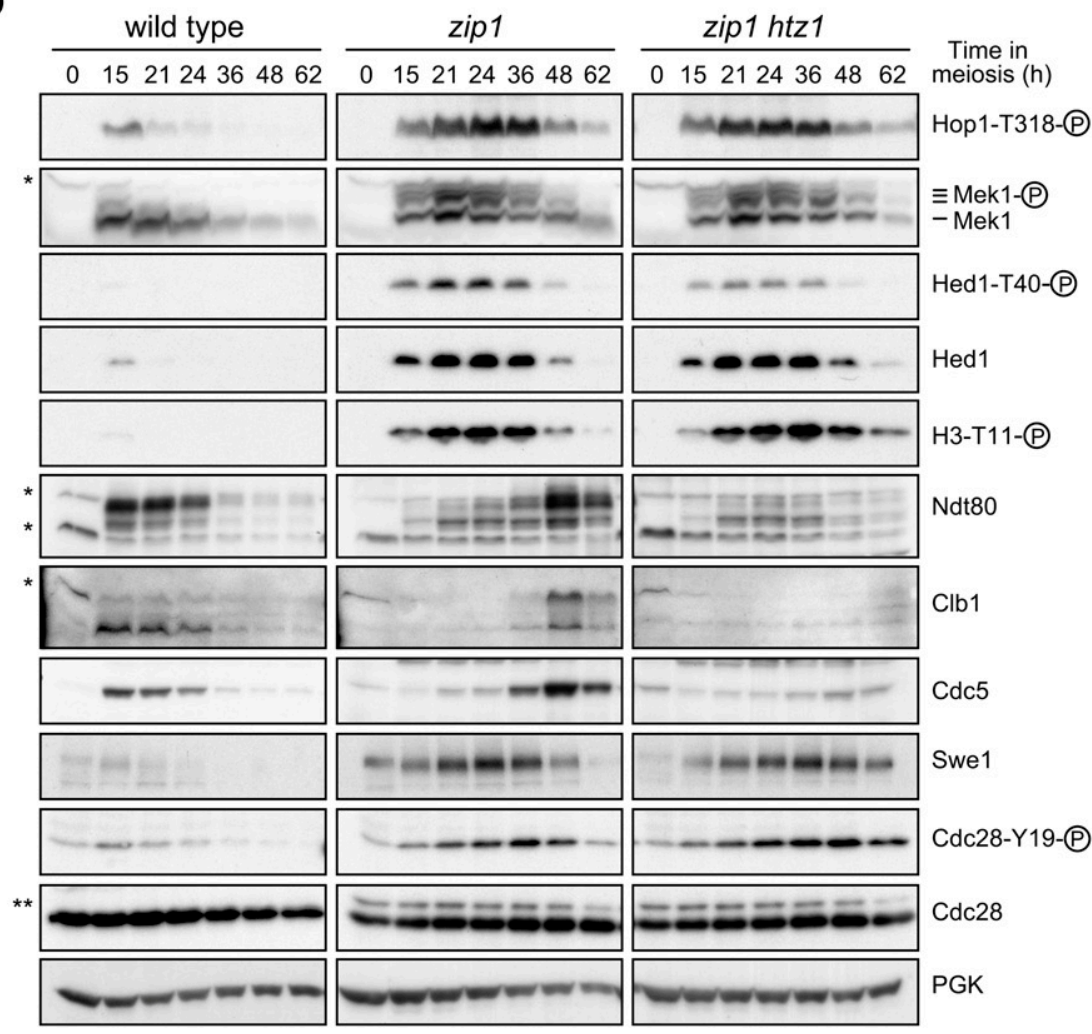

Figure 4 Robust checkpoint-dependent meiotic arrest in zip1 htz1. (A and B) Time course analysis of meiotic nuclear divisions; the percentage of cells containing two or more nuclei is represented. Error bars: SD; $n=3$. Strains are DP421 (wild type), DP422 (zip1), DP776 (zip1 htz1), DP1524 (zip1 spo11), DP815 (zip1 htz1 spo11), and DP816 (zip1 $h t z 1$ ddc2). (C) Localization and quantification of Rad51 foci as markers for unrepaired DSBs on spread meiotic nuclei of zip1 (DP449), zip1 htz1 (DP776), and zip1 htz1 spo11 (DP815) after $16 \mathrm{hr}$ of meiotic induction. Only prophase I nuclei, as assessed by tubulin staining, were scored. Representative images are shown. $* * *, P<0.001$. (D) Western blot analysis of the indicated molecular markers of checkpoint activity at different levels in the pathway. PGK was used as a loading control. Asterisks mark non-specific bands. Phosphorylated forms are indicated by a circled P. Strains are DP421 (wild type), DP422 (zip1), and DP631 (zip1 htz1). For detection of Myc-tagged Swe1, the strains used are DP1353 (wild type), DP1354 (zip1), and DP1414 (zip1 htz1). contrast, we found that although Mek1 was downregulated in zip 1 htz 1 with similar kinetics to that in zip1, Ndt80, Clb1, and Cdc5 production remained largely inhibited, and Swe1 and phospho-Cdc28-Y19 levels stayed high at late time points (Figure 4D), consistent with the inability of zip $1 \mathrm{htz} 1$ cells to exit prophase I (Figure 4A). These results indicate that the main cell cycle targets of the checkpoint are misregulated in the absence of H2A.Z and suggest that this impairment is responsible for the strong block in meiotic progression of zip1 htz1.

\section{HA2.Z contribution to checkpoint recovery}

To determine whether H2A.Z is required to restart meiotic cell cycle progression when the zip 1 defects that initially 
triggered the checkpoint are corrected, we used a conditional system in which ZIP1-GFP expression is controlled by $\beta$ estradiol. ZIP1-GFP was placed under control of the GAL1 promoter in strains producing a version of the Gal4 transcriptional regulator fused the $\beta$-estradiol receptor (Gal4[848]. ER) (Benjamin et al. 2003; Voelkel-Meiman et al. 2012). As depicted in Figure 5A, meiotic cultures of both wild-type and $h t z 1$ strains were initiated without $\beta$-estradiol; that is, in the absence of Zip1, to induce the checkpoint response. After $24 \mathrm{hr}$, when the cells are blocked in prophase by the checkpoint, $\beta$-estradiol was added to half of the culture and the other half was maintained in the absence of the hormone as control. Recovery from the arrest after ZIP1 induction was monitored at the cytological level (Zip1-GFP chromosome incorporation and DAPI staining of nuclei) and at the molecular level (Western blot analysis of various checkpoint markers) (Figure 5, B-D).

In the absence of $\beta$-estradiol ("ZIP1 OFF"), the checkpoint was activated in the wild type as shown by the prominent H3T11 and Hed1-T40 phosphorylation, but eventually the phosphorylation of these markers decreased concomitant with Ndt80 activation, Cdc5 production, and Cdc28-Y19 dephosphorylation (Figure 5D), thus sustaining meiotic progression (Figure 5C). Note that for unknown reasons, the meiotic delay induced by the checkpoint in this ZIP1 OFF situation is less pronounced than in a zip1 mutant (Figure 4A), perhaps due to a leaky but undetectable expression of GAL1-ZIP1 even in the absence of $\beta$-estradiol. In the $h t z 1$ mutant without $\beta$-estradiol, the checkpoint was also heavily activated but, with slightly slower kinetics, the levels of H3-T11 and Hed1-T40 phosphorylation were also finally reduced. However, like in zip $1 \mathrm{htz} 1$ mutants, Ndt80 production was not induced and Cdc28-Y19 remained phosphorylated at late points (Figure 5D) and as a consequence, meiotic progression was robustly blocked (Figure 5C). Thus, this ZIP1 OFF situation phenocopies ZIP1 deletion in $h t z 1$ (Figure 4, A-D).

When $\beta$-estradiol was added, ZIP1-GFP expression was induced, and $3 \mathrm{hr}$ after hormone addition, Zip1-containing chromosomes were detected in nuclei of both wild type and htz1 (Figure 5B). ZIP1-GFP induction was slightly less efficient in the $h t z 1$ mutant (Figure 5D), perhaps due to the effect of H2A.Z on GAL1 promoter regulation (Santisteban et al. 2000). In the wild type, the checkpoint was rapidly turned off upon Zip1 production: Mek1 signaling drastically disappeared, Ndt80 and Cdc5 were sharply induced and Cdc28-Y19 phosphorylation was erased (Figure 5D; "ZIP1 ON"). Consistently, prophase-arrested wild-type cells immediately underwent meiotic divisions after ZIP1 expression (Figure 5C; ZIP1 ON). In the $h t z 1$ mutant the checkpoint was also downregulated upon ZIP1 induction, but with a slower kinetics than that of the wild type. Consistently, a fraction of $h t z 1$ cells resumed meiotic divisions (Figure 5C; ZIP1 ON); thus, H2A.Z is not essential to restart meiotic cell cycle progression when the defects that triggered the checkpoint are resolved, but contributes to an efficient recovery from the cell cycle arrest.

\section{NDT80 overexpression partially alleviates zip1 htz1 meiotic arrest}

Since zip $1 \mathrm{htz} 1$ shows a dramatic reduction in Ndt80 levels and Cdc5 production is also impaired (Figure 4D), we examined whether an artificial increase in CDC5 and NDT80 expression could restore meiotic progression in zip $1 \mathrm{htz} 1$. As reported (Acosta et al. 2011), CDC5 overexpression from a high-copy plasmid partially suppressed the meiotic delay of the zip1 single mutant (Figure 6A); however, it had little effect on zip1 htz 1 (Figure 6B). In contrast, NDT80 overexpression did promote more efficient meiotic progression in both zip1 and zip1 $h t z 1$ (Figure 6, A and B). These observations indicate that, in part, the strong meiotic block of the zip1 htz 1 mutant results from the drastic reduction in Ndt80 production and suggest that, in addition to $C D C 5$, Ndt80 likely targets another factor relevant to promote chromosome segregation in sip $1 \mathrm{htz} 1$.

\section{Deletion of SWE1, but not mutation of Cdc28-Y19, suppresses the zip1 htz1 meiotic block}

We have found that the levels of both the Swe1 kinase and the phosphorylation of its target, Cdc28-Y19, remain high at late time points in the zip $1 \mathrm{htz} 1$ meiotic cultures. To assess the relevance of Cdc28-Y19 inhibitory phosphorylation to impose the tight zip1 htz1 meiotic arrest (Figure 7A), we generated three situations in which this phosphorylation event is either abolished or drastically reduced (Figure 7B): (1) SWE1 deletion, (2) cdc28$A F$ mutation (carrying the threonine 18 and tyrosine 19 of $\mathrm{Cdc} 28$ changed to alanine and phenylalanine, respectively), and (3) overexpression of the MIH1 gene from the prophase I-specific HOP1 promoter in a high-copy plasmid (Figure S6A).

Remarkably, deletion of SWE1 conferred a notable suppression of the zip $1 \mathrm{htz} 1$ meiotic arrest (Figure 7C), although it did not reach wild-type kinetics; however, the elimination of Cdc28-Y19 phosphorylation by other means, such as Mih1 overproduction or $c d c 28-A F$ mutation, had none or only a subtle effect on meiotic progression as most cells remained uninucleated (Figure 7C), with only $\sim 10 \%$ of zip $1 \mathrm{htz} 1$ cdc28-AF cells segregating their nuclei. In contrast, MIH1 overexpression or $c d c 28-A F$ mutation did accelerate meiotic progression in a zip 1 single mutant (Figure S6B). A kinasedead swe1-N584A allele (Harvey et al. 2005) conferred the same suppression of the checkpoint meiotic arrest as the SWE1 deletion both in zip1 and zip1 htz1 strains (Figure $\mathrm{S6C})$, ruling out the possibility of a direct inhibitory effect exerted by the physical interaction of Swe1 with CDK independent of Tyr19 phosphorylation. Thus, these results strongly suggest that the Swe1 kinase must affect an additional mechanism, independent of CDK phosphorylation, which is particularly relevant in the absence of H2A.Z to maintain the zip1-induced checkpoint arrest.

\section{CLB1 overexpression restores meiotic progression in zip1 htz1 cdc28-AF}

To further explore the checkpoint response in zip $1 \mathrm{htz} 1$ and the effect of CDK phosphorylation, we used Western blotting to analyze various molecular markers in the swe 1 and 
A
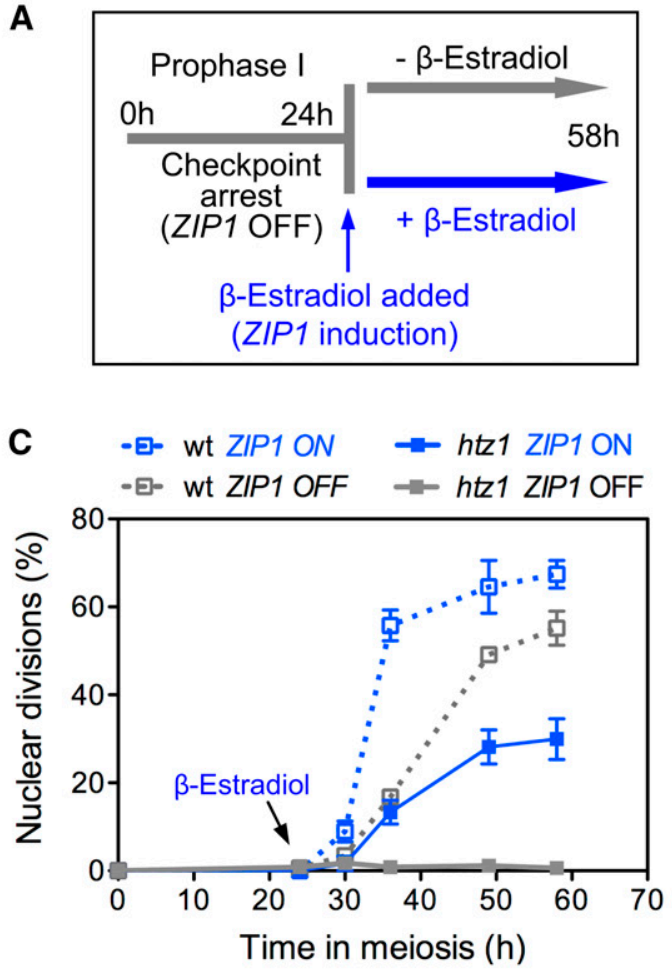

D

\begin{tabular}{|c|c|}
\hline $\begin{array}{c}-\beta \text {-Estradiol } \\
\text { (ZIP1 OFF) }\end{array}$ & $\begin{array}{c}+\beta \text {-Estradiol } \\
(\text { ZIP1 ON })\end{array}$ \\
\hline
\end{tabular}

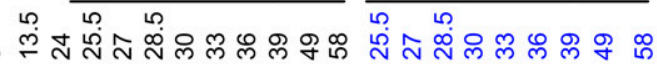
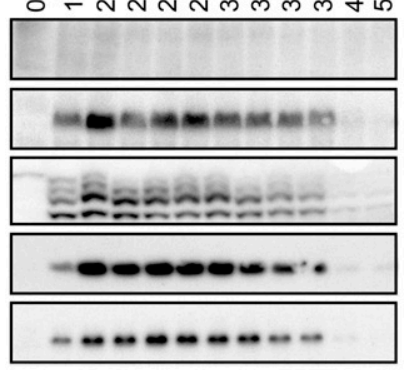

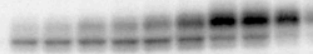

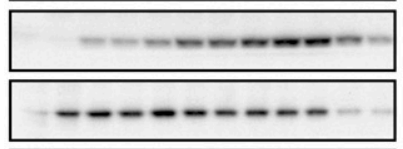

-n-w- - -
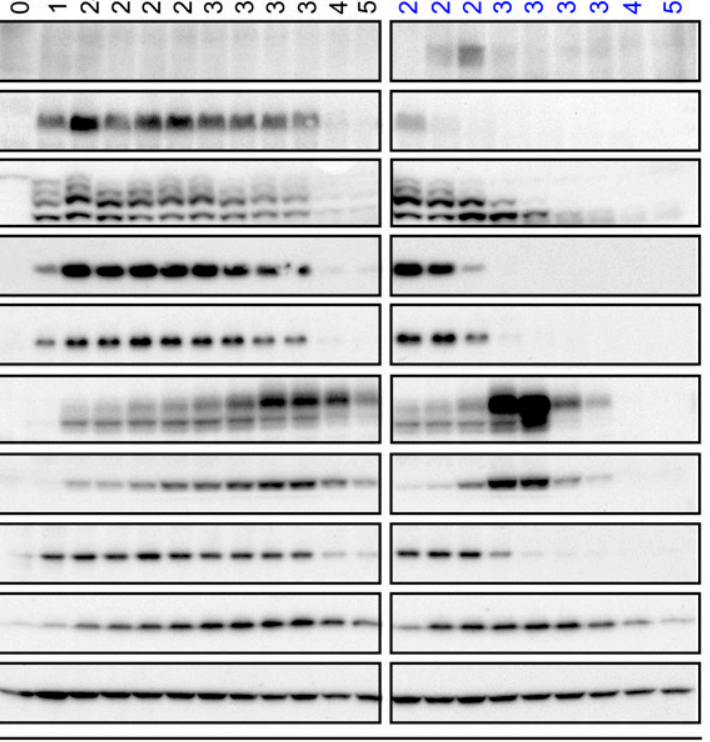

wild type
B

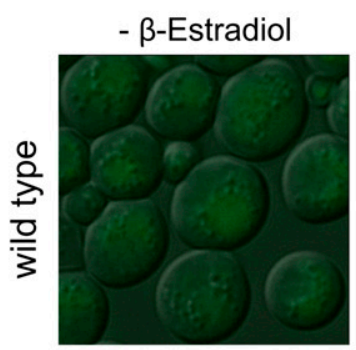

$+\beta$-Estradiol
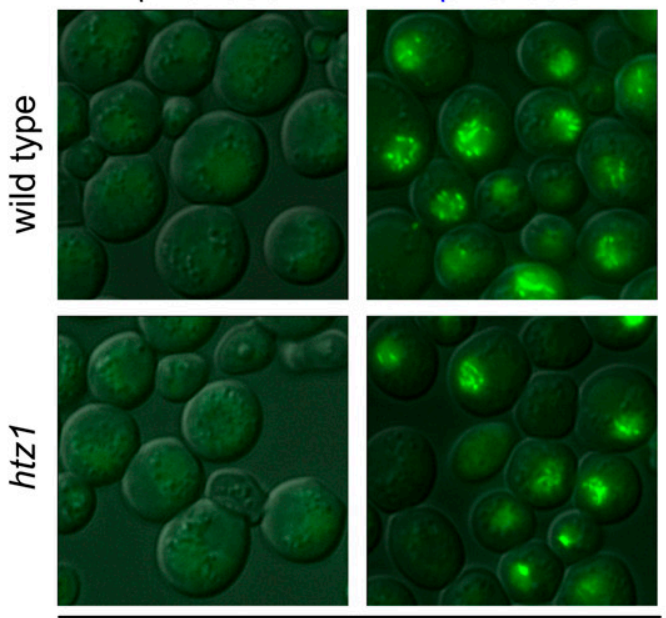

Zip1-GFP $\frac{\begin{array}{c}-\beta \text {-Estradiol } \\ \text { (ZIP1 OFF) }\end{array}}{n} \frac{\begin{array}{c}\beta \text {-Estradiol } \\ \text { (ZIP1 ON) }\end{array}}{\omega}$

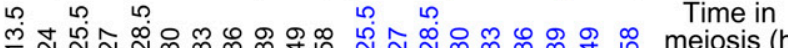
meiosis $(h)$

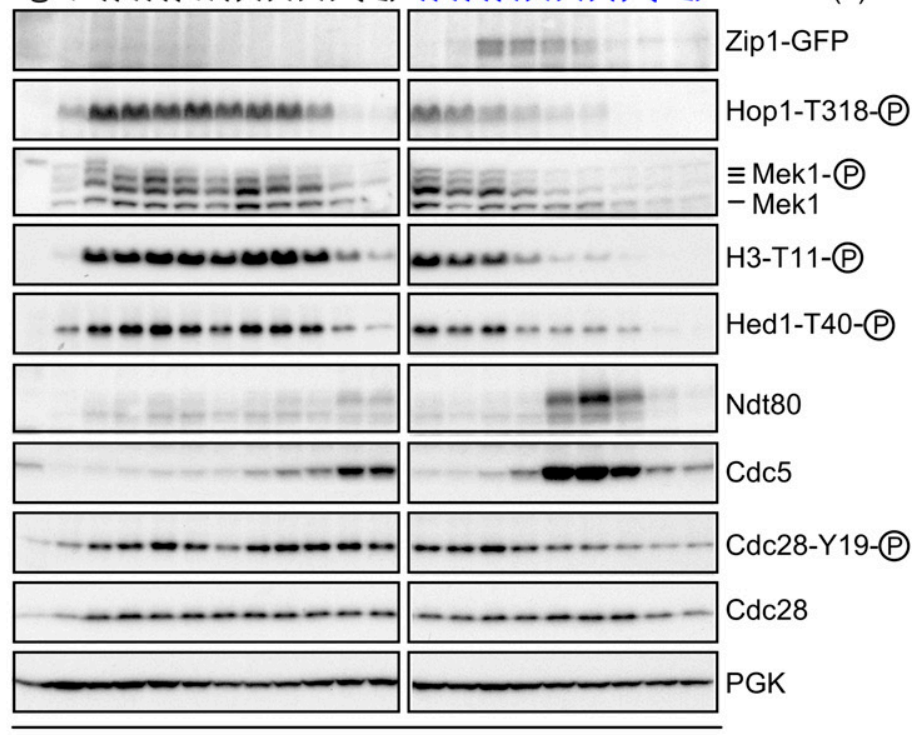

$h t z 1$

Figure 5 Analysis of meiotic checkpoint recovery. (A) Schematic representation of the experimental setup for conditional ZIP1 induction in wild type (DP1185) and $h t z 1$ (DP1186) cells containing the GAL4-ER transcriptional activator regulated by $\beta$-estradiol and $P_{\text {GAL1-ZIP1-GFP. (B) Representative }}$ fluorescence microscopy images showing SC incorporation of Zip1-GFP. Cells were imaged $3 \mathrm{hr}$ after $\beta$-estradiol addition. (C) Time course analysis of meiotic nuclear divisions; the percentage of cells containing two or more nuclei is represented. The arrow indicates $\beta$-estradiol addition (blue lines and symbols). Error bars: range; $n=2$. (D) Western blot analysis of the indicated molecular markers of checkpoint activity. PGK was used as a loading control. wt, wild type. Phosphorylated forms are indicated by a circled P. 


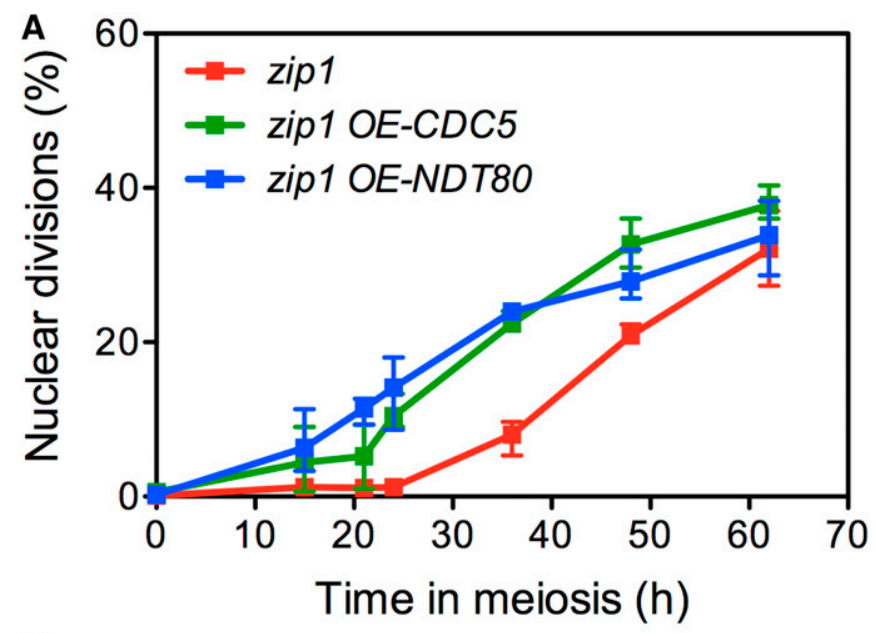

B

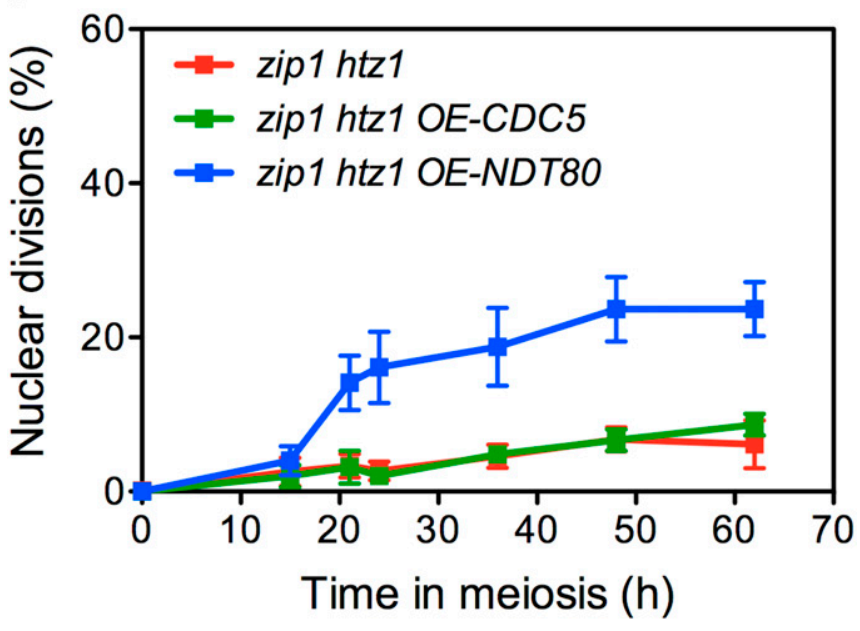

Figure 6 NDT80 overexpression partially suppresses zip1 htz1 meiotic arrest. ( $A$ and $B$ ) Time course analysis of meiotic nuclear divisions; the percentage of cells containing two or more nuclei is represented. Strains are DP422 (zip1) in A and DP1017 (zip1 htz1) in B, transformed with vector alone (pRS426) or with high-copy plasmids overexpressing CDC5 (pJC29) or NDT80 (pSS263), denoted as OE-CDC5 and OE-NDT80, respectively. Error bars: $S D ; n=3$.

$c d c 28-A F$ mutants. According with its meiotic progression (Figure 7C), the checkpoint was deactivated in zip $1 \mathrm{htz} 1$ swe1, as manifested by the disappearance of phospho-Hop1T318 and phospho-Hed1-T40. Concurrently, the meiosis I-promoting factors Ndt80, Clb1, and Cdc5 were produced, albeit with slower kinetics than in the wild type (Figure 7D).

Like in zip1 htz1, upstream checkpoint signals were also downregulated in zip1 htz1 cdc28-AF; in contrast, Ndt80, $\mathrm{Clb} 1$, and Cdc5 accumulated at higher levels at later time points in this mutant (Figure 7D and Figure S6D). The presence of meiosis I-promoting factors suggests that the zip1 $h t z 1 c d c 28-A F$ triple mutant is proficient to undergo the prophase to meiosis I transition, but does not efficiently complete chromosome segregation. Indeed, $\sim 40 \%$ of zip 1 htz 1 cdc28$A F$ cells assembled meiotic spindles at late time points (Figure 7E) despite their marked impairment to undergo meiotic divisions (Figure 7C).
Notably, CLB1 overexpression from a high-copy plasmid restored substantial meiotic progression in zip $1 \mathrm{htz} 1 \mathrm{cdc} 28$ $A F$ phenocopying zip 1 htz 1 swel (Figure 7, C and D). In sum, these observations suggest that, in addition to phosphorylate Cdc28 at tyrosine 19 to prevent exit from prophase I, Swe1 regulates timing and/or abundance of $\mathrm{Clb} 1$ production to restrain meiotic progression in zip $1 \mathrm{htz} 1$ at a later stage in meiotic development.

\section{Discussion}

The H2A.Z histone variant is a ubiquitous determinant of chromatin structure and plays crucial roles in genome stability and gene expression in mitotically dividing eukaryotic cells. However, only a limited number of studies in a few model organisms have addressed the relevance of H2A.Z in meiosis, often using indirect approaches. In this article, we have focused on the direct functional contribution of H2A.Z during meiosis in the budding yeast $S$. cerevisiae, a widely used model system for meiotic studies.

\section{H2A.Z is required for proper meiotic development}

We report here that the $h t z 1$ mutant of $S$. cerevisiae lacking the H2A.Z histone completes the meiotic program, albeit less efficiently than the wild type. The $h t z 1$ mutant shows delayed entry into meiosis, impaired sporulation, and reduced spore viability, indicating that $\mathrm{H} 2 \mathrm{~A} . \mathrm{Z}$ is required to sustain accurate meiosis. The persistence of recombination intermediates or incomplete synapsis triggers the MRC that delays meiotic progression. We found that checkpoint elimination by deleting MEK1 or abolishing DSB formation by deleting SPO11 do not restore normal levels of meiotic nuclear divisions in $h t z 1$, indicating that the faulty events resulting in impaired completion of meiotic development are not sensed by the MRC and likely do not involve recombination.

In fission yeast, H2A.Z participates in the initiation of meiotic recombination by promoting the association of Spo11 and accessory proteins to chromatin (Yamada et al. 2018). We have found a modest reduction in the number of Rad51 foci in zip1 htz1 compared to zip1 (Figure 4C) that could be compatible with reduced number of initiating DSBs, although a slightly defective loading of Rad51 to DSBs in the absence of H2A.Z or a delayed onset of DSB formation cannot be ruled out. A possible role for H2A.Z in DSB generation could be also inferred from the presence of H2A.Z at promoters (at least in vegetative cells) (Raisner et al. 2005) where most DSBs occur in S. cerevisiae (Pan et al. 2011). However, our results suggest that, in budding yeast, the functional contribution of H2A.Z to DSB formation, if any, is only minor: (1) dynamics of Hop1 phosphorylation at T318, which serves as an indirect reporter for meiotic DSBs, is similar in wild type and $h t z 1$; (2) a reduction in DSB formation provoked by the absence of H2A.Z would result in a less stringent checkpoint response, although the zip 1 htz 1 double mutant displays a more robust checkpoint arrest compared to zip 1; (3) crossover recombination in a particular interval of 
A

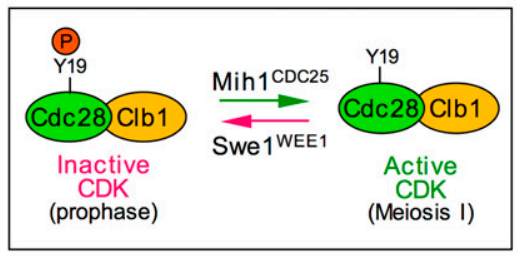

B

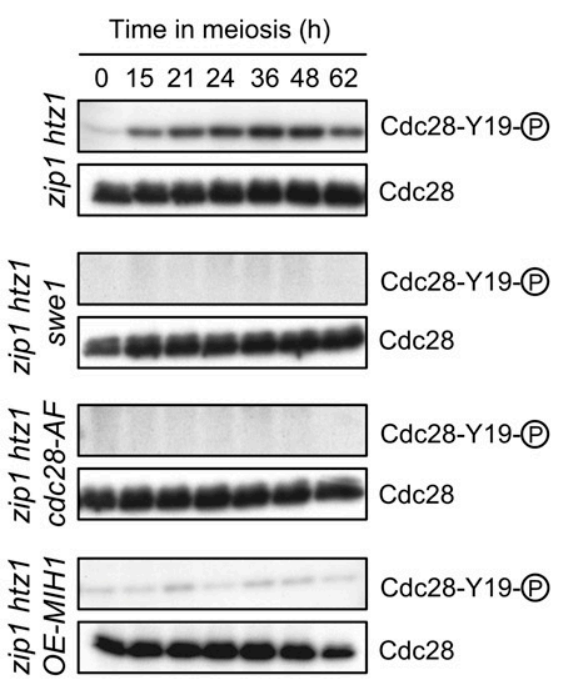

C

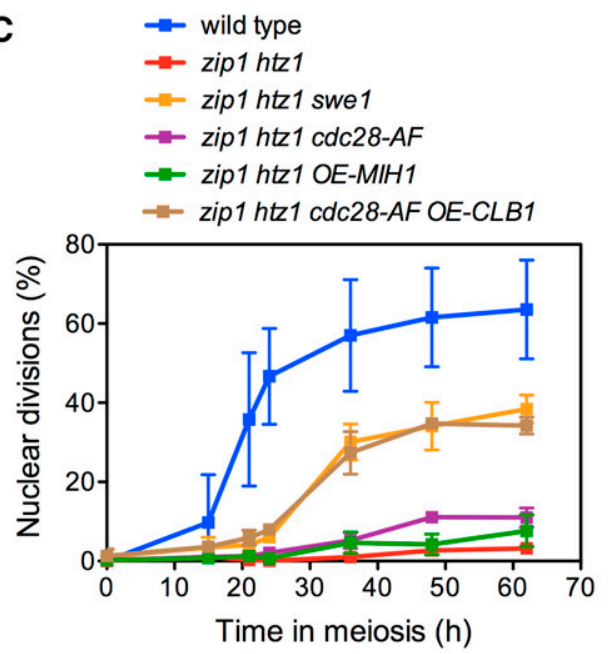

E

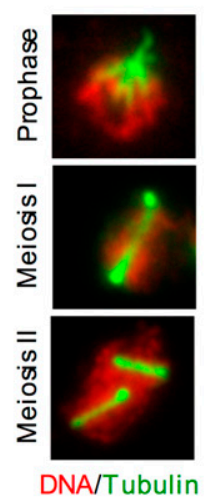

Figure 7 Impact of Cdc28-Y19 phosphorylation and Clb1 levels on zip1 $h t z 1$ meiotic arrest. (A) Schematic representation of the regulation of CDK activity by Cdc28-Y19 phosphorylation controlled by the opposite action of the Swe1 kinase and the Mih1 phosphatase. (B) Western blot analysis of Cdc28-Y19 phosphorylation in the indicated strains. Total Cdc28 is also shown as control. (C) Time course analysis of meiotic nuclear divisions; the percentage of cells containing two or more nuclei is represented. Error bars: SD; $n=3$. (D) Western blot analysis of the indicated molecular markers of checkpoint activity. Swe 1 was detected with anti-myc antibodies. PGK was used as a loading control. Strains in B-D are: DP1353 (wild type), DP1414 (zip1 htz1), DP1113 (zip1 htz1 swe1), and DP1416 (zip1 htz1 cdc28AF). To overexpress $M I H 1$ and $C L B 1$, the DP1414 and DP1416 strains were transformed with high-copy plasmids pSS265 (OE-MIH1) and pR2045 (OE-CLB1), respectively. (E) Whole-cell immunofluorescence using anti-tubulin antibodies in zip 1 htz1 (DP1017) and zip1 htz1 cdc28AF (DP1154) cells at $48 \mathrm{hr}$ in meiosis. Representative nuclei of prophase, meiosis I, and meiosis II stages are shown. The quantification is presented in the graph. 169 and 119 nuclei were scored for zip 1 htz1 and zip1 htz1 cdc28-AF, respectively. Error bars: range; $n=2$.
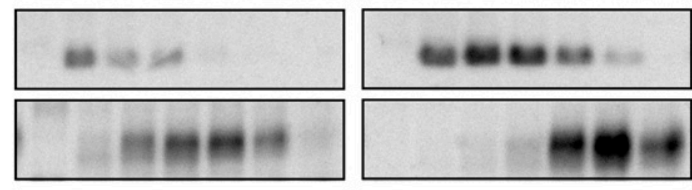

\begin{tabular}{lllllll}
$c$ & \multicolumn{1}{c}{$c$} & $28-A F$ & $O E-C L B 1$ \\
\hline 0 & 15 & 21 & 24 & 36 & 48 & 62
\end{tabular}

Time in
meiosis (h)
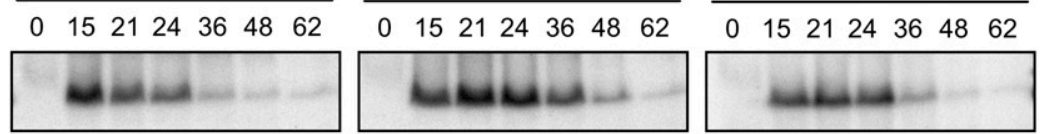

Hop1-T318-巴

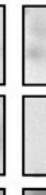
Hed1-T40-P
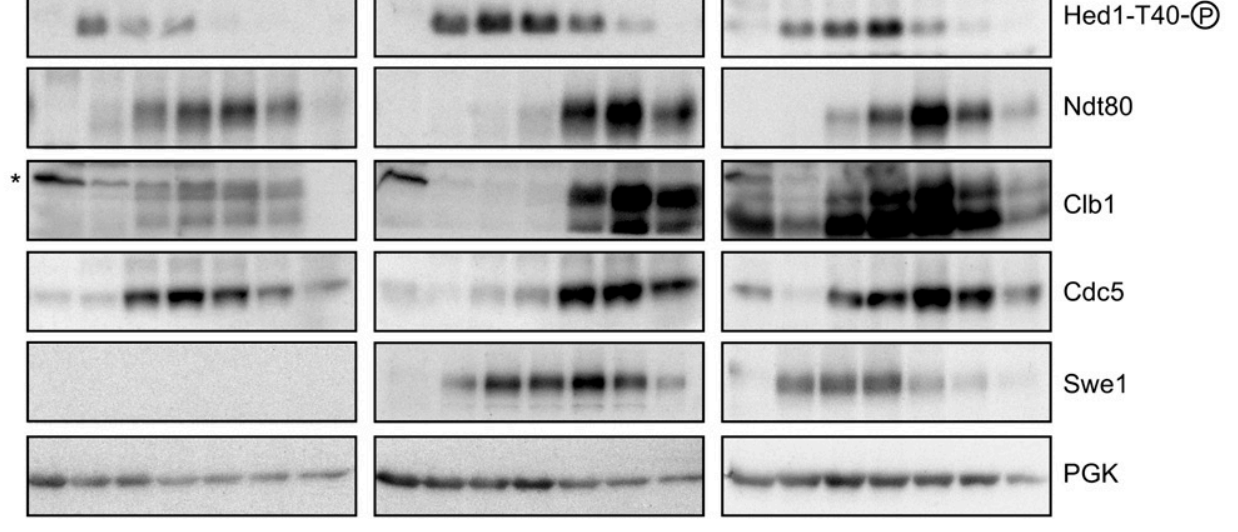

chromosome VIII is not significantly affected by htz1. It is formally possible that recombination could be altered in other chromosomal regions and/or that CO homeostasis could compensate for a reduced number of initiating events (Martini et al. 2006), but this would imply at best a subsidiary function for H2A.Z in DSB formation. In sum, we do not favor the scenario in which the meiotic phenotypes of the htz1 mutant could be solely explained by impaired DSB formation. Our genome-wide study of meiotic gene expression in the $h t z 1$ mutant reveals that many downregulated genes cluster 
in several functional categories related to mitotic and meiotic cell cycle and chromosome segregation events (Table 1 and Table S4). We propose that, in unperturbed conditions, H2A. $\mathrm{Z}$ is not essential to perform any critical meiotic event, but the massive transcription misregulation that occurs in the absence of this histone variant may affect various processes, resulting in a less accurate and efficient completion of the meiotic program.

\section{$H 2 A . Z$ is essential to resume meiotic progression in the absence of Zip1}

Certain chromatin modifications are crucial for checkpoint activity. Dot1-mediated trimethylation of H3K79 controls Pch2 chromosomal distribution and sustains Hop1 phosphorylation and the ensuing Mek1 activation in zip 1 mutants. As a consequence, deletion of DOT1 or mutation of H3K79 suppresses the meiotic arrest/delay of zip1 (San-Segundo and Roeder 2000; Ontoso et al. 2013). The Sir2 histone deacetylase is also essential for the zip1-induced MRC. One of the main targets of Sir2 is acetylated H4K16. In zip 1 sir 2 mutants, as well as in zip1 H4-K16Q mutants (mimicking constitutive H4K16 acetylation), the zip 1 block is bypassed (San-Segundo and Roeder 1999; Cavero et al. 2016). At least in vegetative cells, Dot1 and the SIR complex collaborate with H2A.Z in delimiting the boundaries between euchromatin and telomeric heterochromatin (Dhillon and Kamakaka 2000; Meneghini et al. 2003). However, these chromatin modifications perform opposite functions in the MRC; while the meiotic delay is suppressed in zip 1 dot 1 and zip 1 sir2, zip 1 htz 1 shows a stronger meiotic arrest. Our results imply that, in contrast to Dot1 and Sir2, H2A.Z is not required for checkpoint activation, but it is involved in regulation meiotic progression at least in a zip1 mutant.

We show that the zip 1 mutant exhibits a pronounced meiotic delay, but eventually checkpoint signaling declines, as manifested by the drop in Hop1 phosphorylation and in Mek1 activation at late time points, and at least a fraction of the culture resumes meiotic progression and completes sporulation. In principle, checkpoint deactivation and resumption of cell cycle progression can occur by two related but conceptually different phenomena: "checkpoint adaptation" and "checkpoint recovery." Adaptation takes place when, despite the persistence of the defects that initially triggered the checkpoint, its activity declines after a prolonged period and the cell cycle resumes without previous elimination of the damage. This process of adaptation has been extensively documented in vegetative budding yeast responding to the presence of an irreparable DSB (Pellicioli et al. 2001). In contrast, checkpoint recovery involves the disappearance or repair of the initial problems that stimulated the checkpoint, resulting in decreased signaling and cell cycle progression.

Previous studies suggest that the eventual checkpoint deactivation and recovery of meiotic progression in zip 1 is consequence of the disappearance of the initial defects (likely unrepaired DSBs), presumably by using the sister chromatid instead of the homolog as template for DNA repair. This is based on the observation that deletion of $R A D 51$, which fundamentally compromises sister chromatid recombination (Liu et al. 2014; Callender et al. 2016), leads to a permanent arrest in zip1 (Herruzo et al. 2016) (Figure S7A). In this work we report that, like zip 1 rad51, the zip 1 htz1 double mutant also shows a tight meiotic block; however, the analysis of various checkpoint markers reveals that the cause of the arrest is different in zip 1 rad51 and zip1 htz1. In the zip1 rad51 mutant, high levels of Hop1-T318 phosphorylation and Mek1 activity persist until late time points, consistent with the accumulation of unrepaired recombination intermediates that signal to the checkpoint. Consequently, Cdc28-Ty19 phosphorylation remains high and Cdc5 production is inhibited, thus explaining the meiotic arrest (Herruzo et al. 2016) (Figure S7B). In contrast, we show that in zip 1 htz1, Hop 1 and Mek1 activation eventually decline with similar kinetics to that observed in the zip1 single mutant, although meiosis I-promoting factors (i.e., Ndt80, Cdc28, Cdc5, and Clb1) remain largely inhibited. These observations imply that the disappearance of the initial signal stimulating the checkpoint is not affected by $h t z 1$, placing H2A.Z function downstream in the pathway.

\section{Influence of H2A.Z on Ndt80 and CDK activity}

In our molecular analysis of the zip1-induced MRC pathway at various levels, the main alterations detected resulting from the absence of H2A.Z were the dramatic reduction in Ndt80 levels and the persistence of both the Swe1 kinase and phosphorylation of its substrate Cdc18-Y19. The observation that NDT80 overexpression partially suppresses the zip 1 htz 1 arrest raises the possibility that $\mathrm{H} 2 \mathrm{~A}$. $\mathrm{Z}$ could be directly or indirectly controlling NDT80 gene expression. It has been recently described that Bdf1, a subunit of the SWR1 complex involved in the interaction with certain histone marks at particular nucleosomes (Altaf et al. 2010), is required for meiotic progression and sporulation. Bdf1 binds to the NDT80 promoter through the BD1 and BD2 bromodomains promoting its transcription (Garcia-Oliver et al. 2017). Nevertheless, several observations suggest that H2A.Z does not control Ndt80 levels via Bdf1. The interaction of Bdf1 with the NDT80 promoter is independent of the SWR1 complex (Garcia-Oliver et al. 2017), consistent with our observation that meiotic progression is not significantly affected in the swr 1 single mutant (Figure S2, A and B). However, the meiotic checkpoint function of H2A.Z does depend on SWR1 since both zip 1 htz1 and zip1 swr1 show meiotic arrest (Figure S4C). In addition, strong BDF1 overexpression does not promote sporulation in zip 1 htz1 (Figure S8A). Moreover, we did not find a significant change in NDT80 transcript levels in our genome-wide expression analysis of the $h t z 1$ mutant during meiosis. Regulation of NDT80 expression is quite complex and also involves the elimination of the Sum 1 repressor binding to the middle-sporulation elements in its promoter. The displacement of Sum 1 from the middle-sporulation elements requires the competition with $\mathrm{Ndt} 80$ and also the phosphorylation of Sum 1 by Ime 2 and CDK (Winter 2012). We found 


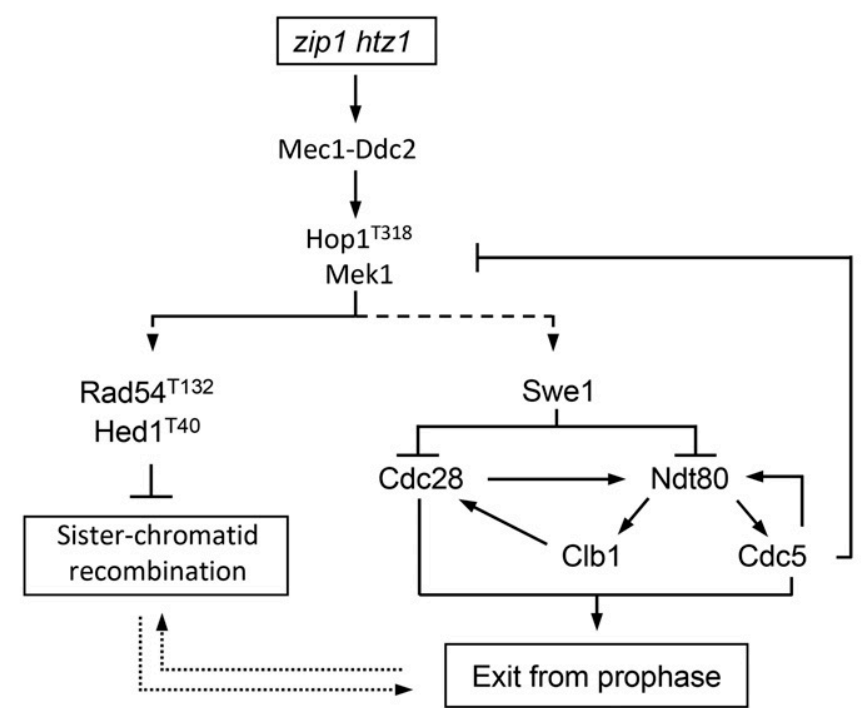

B $_{\text {zip1 htz1 OE-NDT80 }}$
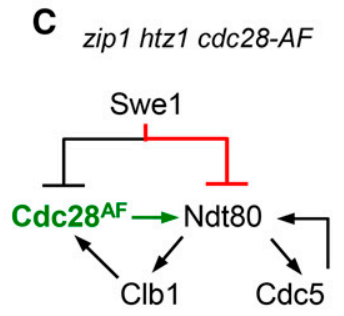

Impaired meiotic progression

D zip1 htz1 swe1

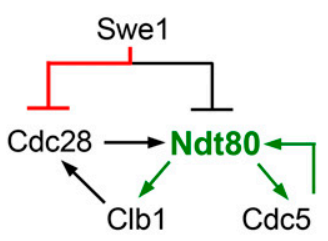

Partial suppression of meiotic block
Figure 8 Exit from prophase I in S. cerevisiae. (A) A model for the regulation of the prophase to meiosis I transition by the meiotic recombination checkpoint. See discussion for details. The discontinuous line connecting Mek1 and Swe1 indicates that there is no evidence for direct phosphorylation of Swe1 by Mek1. A functional connection or dependency between DSB repair by sister chromatid recombination and entry into meiosis I is represented by dotted lines. (B-D) The impact on meiotic progression resulting from the mutant conditions indicated. Green and red colors represent the predominant positive and negative effects, respectively. that, like zip 1 htz1, the zip 1 htz 1 sum 1 triple mutant remains blocked in meiosis (Figure S8B), indicating that H2A.Z does not exert its effect on Ndt80 levels via Sum1. In addition, activation of Ndt80 requires its phosphorylation in the nucleus; stimulation of the MRC results in cytoplasmic sequestration of Ndt80 (Wang et al. 2011). It is tempting to speculate that H2A.Z could be involved, directly or indirectly, in the nuclear import of Ndt80 when the signal stimulating the checkpoint by the absence of Zip1 declines. The contribution of H2A.Z to the nuclear transport of other proteins has been reported in yeast (Gardner et al. 2011), but the almost undetectable levels of Ndt80 in zip 1 htz 1 complicate this analysis with the tools currently available.

Our results also show that, in zip $1 \mathrm{htz} 1$, Swe1-dependent inhibitory phosphorylation of Cdc28-Y19 persists longer than in zip1, suggesting that H2A.Z action may be impinging on CDK activity. In fact, deletion of SWE1, which abolishes Cdc29-Y19 phosphorylation, significantly suppresses zip1 htz1 arrest. Since MIH1, the gene encoding the phosphatase that reverts Cdc28-Y19 phosphorylation, was found among the genes whose meiotic expression decreases in the $h t z 1$ mutant (Table 1), it is plausible to postulate that lower levels of the Mih1 phosphatase in zip1 htz1 could explain the accumulation of phosphorylated Cdc28-Y19 and the impaired meiotic progression. However, we demonstrate that strong overproduction of Mih1, which results in negligible Cdc28Y19 levels, does not restore meiotic nuclear divisions in zip1 $h t z 1$. This observation, together with the fact that a nonphosphorylatable $c d c 28-A F$ mutant also has a minimal impact on the kinetics of meiotic progression of zip 1 htz1, strongly suggest that Swe1 must possess another target in addition to CDK to restrain meiosis in zip1 htz1.

Besides CDK, only a limited number of substrates for Swe1/Wee1 have been described. One attractive candidate is Y40 of histone H2B, which is phosphorylated by Swe1 in yeast (or H2B-Y37 phosphorylated by Wee1 in mammals) to control transcription of histone genes (Mahajan et al. 2012). H2A.Z interacts with H2B in the nucleosomes; therefore, it is formally possible that the conformational change induced by SWR1-dependent substitution of histone H2A by H2A.Z could modulate the phosphorylation of H2B-Y40 by Swe1. To explore if this chromatin modification has an impact on the MRC, we have generated and analyzed a nonphosphorylatable $h t b 1$-Y4OF mutant and found that the zip 1 htz1 $h t b 2 \Delta h t b 1-Y 40 F$ mutant displays the same meiotic arrest as zip1 htz1 (Figure S8C), indicating that this additional Swe1 target is not relevant for the checkpoint response.

It is surprising that in the zip 1 htz1 cdc28-AF mutant we observe the induction and accumulation of the proteins involved in meiosis I entry, such as Ndt80, Clb1, and Cdc5, but most cells remain uninucleated (Figure 7). This situation (i.e., accumulation of Ndt80, Cdc5, and Clb1) is reminiscent of the metaphase I arrest induced by a meiotic-depletion $P_{C L B 2}$-cdc20 mutant (Okaz et al. 2012) and suggests that at least some zip1 
$h t z 1$ cdc28-AF cells are capable of exiting prophase and may arrest at a later stage, such as the metaphase to anaphase I transition. Remarkably, CLB1 overexpression in zip1 htz1 cdc28-AF allows completion of meiotic divisions to a similar degree as the zip1 htz1 swe1 mutant. This observation is consistent with the notion that, in the absence of CDK inhibitory phosphorylation (i.e., $c d c 28-A F$ ), Swe1 negatively controls CLB1 levels in zip1 htz1, likely by inhibiting a CLB1-promoting factor. We note that overexpression of CLB1 from a high-copy plasmid not only increases the global amount of Clb1, but also accelerates its production being detected at earlier time points in the meiotic kinetics. Execution of proper prophase to meiosis I transition is under tight temporal control by a number of events, including the sequential degradation and accumulation of mitotic and meiotic factors, respectively (Okaz et al. 2012). We show that CLB1 overexpression in zip 1 htz1 $c d c 28-A F$ partially restores the proper scenario for timely execution of meiotic transitions. Clb1 is phosphorylated in a CDK- and Cdc5-dependent manner and is imported to the nucleus by a mechanism that depends on CDK, but not Cdc5 activity. Although Clb1 nuclear localization is not essential for meiotic nuclear divisions, it contributes to efficient meiosis I exit (Tibbles et al. 2013). On the other hand, the biological relevance of Clb1 phosphorylation remains to be established, but it correlates with the induction of Cdc5. What is the identity of the CLB1-promoting factor negatively controlled by Swe1? We speculate that Swe1 could be acting, directly or indirectly, on Ndt80 to inhibit its activity especially in the absence of H2A.Z. We propose a model in which Swe1 action could affect both CDK and Ndt80 activity to restrain meiotic progression (Figure 8A). A cross-talk between CDK and Ndt80 activation in checkpoint-inducing conditions has been also documented (Acosta et al. 2011). This model would explain the following situations. (1) in the zip1 htz1 mutant overexpressing NDT80, exogenous levels of this transcription factor could partially overcome Swe1 inhibitory effect on Ndt80, resulting only in a partial release of the meiotic arrest (Figure 6) because Swe1-dependent Cdc28-Y19 phosphorylation would persist (Figure 8B); (2) in the zip1 htz1 cdc28-AF, the inhibition of CDK by Swe1 is released because the phosphorylation target is mutated, but the timing of Clb1 induction is incorrect due the opposite effect of CDK and Swe1 on Ndt80 preventing proper meiotic progression (Figure 8C); and (3) in the zip1 htz 1 swe1, both inhibitions on CDK and Ndt80 disappear sustaining meiotic progression (Figure 8D).

In summary, the detailed analysis of the MRC in the zip1 $h t z 1$ has allowed us to discover novel functional interactions between the downstream components of the pathway driving meiotic cell cycle progression. Why are these aspects particularly manifested in the absence of H2A.Z? We show here that a number of genes involved in different cell cycle events are misregulated in the $h t z 1$ mutant. A feasible explanation is that the unbalanced levels of cell cycle regulators creates more stringent conditions for meiosis I entry in zip $1 \mathrm{htz} 1 \mathrm{in}$ comparison with zip1, thus revealing more subtle aspects of the molecular mechanisms regulating meiotic progression when the MRC is deactivated. Additional work will be required to pinpoint the relevant factors targeted by H2A.Z.

\section{Acknowledgments}

We thank Shirleen Roeder, Jacqueline Segall, Nancy Hollingsworth, Scott Keeney, Michael Lichten, Amy Macqueen, Jesús Carballo, Raimundo Freire, and Marisol Santisteban for providing plasmids, strains, and/or antibodies. We are also grateful to Irene Gil-Torres and David Núñez for help in strain construction and analysis, to Isabel Acosta for technical assistance, and to Andrés Clemente-Blanco and José Pérez-Martín for valuable discussion and suggestions. This work was funded by grants BFU2015-63698-P (to F.P.), BFU201565417-R (to P.S.-S.), and BFU2015-69142-REDT (to F.P. and P.S.-S.) from the Ministry of Economy and Competitiveness of Spain (MINECO), and grant CSI084U16 from Junta de Castilla y León (Spain), to P.S.-S.

\section{Literature Cited}

Acosta, I., D. Ontoso, and P. A. San-Segundo, 2011 The budding yeast polo-like kinase Cdc5 regulates the Ndt80 branch of the meiotic recombination checkpoint pathway. Mol. Biol. Cell 22: 3478-3490. https://doi.org/10.1091/mbc.E11-06-0482

Adkins, N. L., H. Niu, P. Sung, and C. L. Peterson, 2013 Nucleosome dynamics regulates DNA processing. Nat. Struct. Mol. Biol. 20: 836-842. https://doi.org/10.1038/nsmb.2585

Altaf, M., A. Auger, J. Monnet-Saksouk, J. Brodeur, S. Piquet et al., 2010 NuA4-dependent acetylation of nucleosomal histones H4 and H2A directly stimulates incorporation of H2A.Z by the SWR1 complex. J. Biol. Chem. 285: 15966-15977. https://doi. org/10.1074/jbc.M110.117069

Argunhan, B., W. K. Leung, N. Afshar, Y. Terentyev, V. V. Subramanian et al., 2017 Fundamental cell cycle kinases collaborate to ensure timely destruction of the synaptonemal complex during meiosis. EMBO J. 36: 2488-2509. https://doi.org/10.15252/ embj.201695895

Benjamin, K. R., C. Zhang, K. M. Shokat, and I. Herskowitz, 2003 Control of landmark events in meiosis by the CDK Cdc28 and the meiosis-specific kinase Ime2. Genes Dev. 17: 1524-1539.

Billon, P., and J. Côté, 2013 Precise deposition of histone H2A.Z in chromatin for genome expression and maintenance. Biochim. Biophys. Acta 1819: 290-302. https://doi.org/10.1016/j. bbagrm.2011.10.004

Bishop, D. K., D. Park, L. Xu, and N. Kleckner, 1992 DMC1: a meiosis-specific yeast homolog of E. coli recA required for recombination, synaptonemal complex formation, and cell cycle progression. Cell 69: 439-456.

Borner, G. V., N. Kleckner, and N. Hunter, 2004 Crossover/ noncrossover differentiation, synaptonemal complex formation, and regulatory surveillance at the leptotene/zygotene transition of meiosis. Cell 117: 29-45.

Brachet, E., V. Sommermeyer, and V. Borde, 2012 Interplay between modifications of chromatin and meiotic recombination hotspots. Biol. Cell 104: 51-69. https://doi.org/10.1111/ boc. 201100113

Callender, T. L., R. Laureau, L. Wan, X. Chen, R. Sandhu et al., 2016 Mek1 down regulates Rad51 activity during yeast meiosis by phosphorylation of Hed1. PLoS Genet. 12: e1006226 (erratum: PLoS Genet. 12: e1006283). https://doi.org/10.1371/ journal.pgen.1006283 
Carballo, J. A., A. L. Johnson, S. G. Sedgwick, and R. S. Cha, 2008 Phosphorylation of the axial element protein Hop1 by Mec1/Tel1 ensures meiotic interhomolog recombination. Cell 132: 758-770. https://doi.org/10.1016/j.cell.2008.01.035

Cavero, S., E. Herruzo, D. Ontoso, and P. A. San-Segundo, 2016 Impact of histone H4K16 acetylation on the meiotic recombination checkpoint in Saccharomyces cerevisiae. Microb. Cell 3: 606-620. https://doi.org/10.15698/mic2016.12.548

Chen, X., R. T. Suhandynata, R. Sandhu, B. Rockmill, N. Mohibullah et al., 2015 Phosphorylation of the synaptonemal complex protein Zip1 regulates the crossover/noncrossover decision during yeast meiosis. PLoS Biol. 13: e1002329. https://doi.org/ 10.1371/journal.pbio.1002329

Choi, K., X. Zhao, K. A. Kelly, O. Venn, J. D. Higgins et al., 2013 Arabidopsis meiotic crossover hot spots overlap with H2A.Z nucleosomes at gene promoters. Nat. Genet. 45: 13271336. https://doi.org/10.1038/ng.2766

Chu, S., J. DeRisi, M. Eisen, J. Mulholland, D. Botstein et al., 1998 The transcriptional program of sporulation in budding yeast. Science 282: 699-705.

Chuang, C. N., Y. H. Cheng, and T. F. Wang, 2012 Mek1 stabilizes Hop1-Thr318 phosphorylation to promote interhomolog recombination and checkpoint responses during yeast meiosis. Nucleic Acids Res. 40: 11416-11427. https://doi.org/10.1093/nar/gks920

Dhillon, N., and R. T. Kamakaka, 2000 A histone variant, Htz1p, and a Sir1p-like protein, Esc2p, mediate silencing at HMR. Mol. Cell 6: 769-780.

Dong, H., and G. S. Roeder, 2000 Organization of the yeast Zip1 protein within the central region of the synaptonemal complex. J. Cell Biol. 148: 417-426.

Garcia-Oliver, E., C. Ramus, J. Perot, M. Arlotto, M. Champleboux et al., 2017 Bdf1 bromodomains are essential for meiosis and the expression of meiotic-specific genes. PLoS Genet. 13: e1006541. https://doi.org/10.1371/journal.pgen.1006541

Gardner, J. M., C. J. Smoyer, E. S. Stensrud, R. Alexander, M. Gogol et al., 2011 Targeting of the SUN protein Mps3 to the inner nuclear membrane by the histone variant H2A. Z. J. Cell Biol. 193: 489-507. https://doi.org/10.1083/jcb.201011017

Goldstein, A. L., and J. H. McCusker, 1999 Three new dominant drug resistance cassettes for gene disruption in Saccharomyces cerevisiae. Yeast 15: 1541-1553.

Greaves, I. K., D. Rangasamy, M. Devoy, J. A. Marshall Graves, and D. J. Tremethick, 2006 The $\mathrm{X}$ and Y chromosomes assemble into H2A.Z-containing facultative heterochromatin following meiosis. Mol. Cell. Biol. 26: 5394-5405.

Harvey, S. L., A. Charlet, W. Haas, S. P. Gygi, and D. R. Kellogg, 2005 Cdk1-dependent regulation of the mitotic inhibitor Wee1. Cell 122: 407-420.

Herruzo, E., D. Ontoso, S. Gonzalez-Arranz, S. Cavero, A. Lechuga et al., 2016 The Pch2 AAA + ATPase promotes phosphorylation of the Hop 1 meiotic checkpoint adaptor in response to synaptonemal complex defects. Nucleic Acids Res. 44: 7722-7741. https://doi.org/10.1093/nar/gkw506

Horigome, C., Y. Oma, T. Konishi, R. Schmid, I. Marcomini et al., 2014 SWR1 and INO80 chromatin remodelers contribute to DNA double-strand break perinuclear anchorage site choice. Mol. Cell 55: 626-639. https://doi.org/10.1016/j.molcel.2014.06.027

Huang, D. W., B. T. Sherman, Q. Tan, J. Kir, D. Liu et al., 2007 DAVID Bioinformatics Resources: expanded annotation database and novel algorithms to better extract biology from large gene lists. Nucleic Acids Res. 35: W169-W175. https:// doi.org/10.1093/nar/gkm415

Hunter, N., 2015 Meiotic recombination: the essence of heredity. Cold Spring Harb. Perspect. Biol. 7: a016618. https://doi.org/ 10.1101/cshperspect.a016618

Joshi, N., M. S. Brown, D. K. Bishop, and G. V. Borner, 2015 Gradual implementation of the meiotic recombination program via checkpoint pathways controlled by global DSB levels. Mol. Cell 57: 797-811. https://doi.org/10.1016/j.molcel.2014.12.027

Kalocsay, M., N. J. Hiller, and S. Jentsch, 2009 Chromosome-wide Rad51 spreading and SUMO-H2A.Z-dependent chromosome fixation in response to a persistent DNA double-strand break. Mol. Cell 33: 335-343. https://doi.org/10.1016/j.molcel.2009. 01.016

Keeney, S., C. N. Giroux, and N. Kleckner, 1997 Meiosis-specific DNA double-strand breaks are catalyzed by Spo11, a member of a widely conserved protein family. Cell 88: 375-384.

Keeney, S., J. Lange, and N. Mohibullah, 2014 Self-organization of meiotic recombination initiation: general principles and molecular pathways. Annu. Rev. Genet. 48: 187-214. https://doi. org/10.1146/annurev-genet-120213-092304

Kniewel, R., H. Murakami, Y. Liu, M. Ito, K. Ohta et al., 2017 Histone H3 threonine 11 phosphorylation is catalyzed directly by the meiosis-specific kinase Mek1 and provides a molecular readout of Mek1 activity in vivo. Genetics 207: 13131333. https://doi.org/10.1534/genetics.117.300359

Kobor, M. S., S. Venkatasubrahmanyam, M. D. Meneghini, J. W. Gin, J. L. Jennings et al., 2004 A protein complex containing the conserved Swi2/Snf2-related ATPase Swr1p deposits histone variant H2A.Z into euchromatin. PLoS Biol. 2: E131.

Krogan, N. J., M. C. Keogh, N. Datta, C. Sawa, O. W. Ryan et al., 2003 A Snf2 family ATPase complex required for recruitment of the histone H2A variant Htz1. Mol. Cell 12: 1565-1576.

Leu, J. Y., and G. S. Roeder, 1999 The pachytene checkpoint in $S$. cerevisiae depends on Swe1-mediated phosphorylation of the cyclin-dependent kinase Cdc28. Mol. Cell 4: 805-814.

Liu, Y., W. A. Gaines, T. Callender, V. Busygina, A. Oke et al., 2014 Down-regulation of Rad51 activity during meiosis in yeast prevents competition with Dmc1 for repair of doublestrand breaks. PLoS Genet. 10: e1004005. https://doi.org/ 10.1371/journal.pgen.1004005

Longtine, M. S., A. McKenzie, III, D. J. Demarini, N. G. Shah, A. Wach et al., 1998 Additional modules for versatile and economical PCR-based gene deletion and modification in Saccharomyces cerevisiae. Yeast 14: 953-961.

MacQueen, A. J., and A. Hochwagen, 2011 Checkpoint mechanisms: the puppet masters of meiotic prophase. Trends Cell Biol. 21: 393-400. https://doi.org/10.1016/j.tcb.2011.03.004

Mahajan, K., B. Fang, J. M. Koomen, and N. P. Mahajan, 2012 H2B Tyr37 phosphorylation suppresses expression of replication-dependent core histone genes. Nat. Struct. Mol. Biol. 19: 930-937. https://doi.org/10.1038/nsmb.2356

Martini, E., R. L. Diaz, N. Hunter, and S. Keeney, 2006 Crossover homeostasis in yeast meiosis. Cell 126: 285-295.

Meneghini, M. D., M. Wu, and H. D. Madhani, 2003 Conserved histone variant H2A.Z protects euchromatin from the ectopic spread of silent heterochromatin. Cell 112: 725-736.

Mizuguchi, G., X. Shen, J. Landry, W. H. Wu, S. Sen et al., 2004 ATP-driven exchange of histone H2AZ variant catalyzed by SWR1 chromatin remodeling complex. Science 303: 343348.

Morillo-Huesca, M., M. Clemente-Ruiz, E. Andujar, and F. Prado, 2010 The SWR1 histone replacement complex causes genetic instability and genome-wide transcription misregulation in the absence of H2A. Z. PLoS One 5: e12143. https://doi.org/ 10.1371/journal.pone.0012143

Niu, H., L. Wan, B. Baumgartner, D. Schaefer, J. Loidl et al., 2005 Partner choice during meiosis is regulated by Hop1-promoted dimerization of Mek1. Mol. Biol. Cell 16: 5804-5818.

Niu, H., L. Wan, V. Busygina, Y. Kwon, J. A. Allen et al., 2009 Regulation of meiotic recombination via Mek1-mediated Rad54 phosphorylation. Mol. Cell 36: 393-404. https://doi. org/10.1016/j.molcel.2009.09.029 
Okaz, E., O. Arguello-Miranda, A. Bogdanova, P. K. Vinod, J. J. Lipp et al., 2012 Meiotic prophase requires proteolysis of $\mathrm{M}$ phase regulators mediated by the meiosis-specific APC/CAma1. Cell 151: 603-618. https://doi.org/10.1016/j.cell.2012.08.044

Ontoso, D., I. Acosta, F. van Leeuwen, R. Freire, and P. A. SanSegundo, 2013 Dot1-dependent histone H3K79 methylation promotes activation of the Mek1 meiotic checkpoint effector kinase by regulating the Hop1 adaptor. PLoS Genet. 9: e1003262. https://doi.org/10.1371/journal.pgen.1003262

Ontoso, D., L. Kauppi, S. Keeney, and P. A. San-Segundo, 2014 Dynamics of DOT1L localization and H3K79 methylation during meiotic prophase I in mouse spermatocytes. Chromosoma 123: 147-164. https://doi.org/10.1007/s00412-013-0438-5

Pak, J., and J. Segall, 2002 Regulation of the premiddle and middle phases of expression of the NDT80 gene during sporulation of Saccharomyces cerevisiae. Mol. Cell. Biol. 22: 6417-6429.

Pan, J., M. Sasaki, R. Kniewel, H. Murakami, H. G. Blitzblau et al., 2011 A hierarchical combination of factors shapes the genomewide topography of yeast meiotic recombination initiation. Cell 144: 719-731. https://doi.org/10.1016/j.cell.2011.02.009

Pellicioli, A., S. E. Lee, C. Lucca, M. Foiani, and J. E. Haber, 2001 Regulation of Saccharomyces Rad53 checkpoint kinase during adaptation from DNA damage-induced G2/M arrest. Mol. Cell 7: 293-300.

Penedos, A., A. L. Johnson, E. Strong, A. S. Goldman, J. A. Carballo et al., 2015 Essential and checkpoint functions of budding yeast ATM and ATR during meiotic prophase are facilitated by differential phosphorylation of a meiotic adaptor protein, Hop1. PLoS One 10: e0134297 [corrigenda: PLoS One11: e0154170 (2016)]. https://doi.org/10.1371/journal. pone.0134297

Prugar, E., C. Burnett, X. Chen, and N. M. Hollingsworth, 2017 Coordination of double strand break repair and meiotic progression in yeast by a Mek1-Ndt80 negative feedback loop. Genetics 206: 497-512. https://doi.org/10.1534/genetics.117.199703

Qin, Y., L. Zhao, M. I. Skaggs, S. Andreuzza, T. Tsukamoto et al., 2014 Actin-related protein6 regulates female meiosis by modulating meiotic gene expression in arabidopsis. Plant Cell 26: 1612-1628.

Raisner, R. M., P. D. Hartley, M. D. Meneghini, M. Z. Bao, C. L. Liu et al., 2005 Histone variant H2A.Z marks the $5^{\prime}$ ends of both active and inactive genes in euchromatin. Cell 123: 233-248.

Refolio, E., S. Cavero, E. Marcon, R. Freire, and P. A. San-Segundo, 2011 The Ddc2/ATRIP checkpoint protein monitors meiotic recombination intermediates. J. Cell Sci. 124: 2488-2500. https://doi.org/10.1242/jcs.081711

Rockmill, B., 2009 Chromosome spreading and immunofluorescence methods in Saccharomyes cerevisiae. Methods Mol. Biol. 558: 3-13. https://doi.org/10.1007/978-1-60761-103-5_1

Rockmill, B., and G. S. Roeder, 1990 Meiosis in asynaptic yeast. Genetics 126: 563-574.

San-Segundo, P. A., and G. S. Roeder, 1999 Pch2 links chromatin silencing to meiotic checkpoint control. Cell 97: 313-324.

San-Segundo, P. A., and G. S. Roeder, 2000 Role for the silencing protein Dot1 in meiotic checkpoint control. Mol. Biol. Cell 11: 3601-3615.

Santisteban, M. S., T. Kalashnikova, and M. M. Smith, 2000 Histone H2A.Z regulats transcription and is partially redundant with nucleosome remodeling complexes. Cell 103: 411-422.

Sheff, M. A., and K. S. Thorn, 2004 Optimized cassettes for fluorescent protein tagging in Saccharomyces cerevisiae. Yeast 21: 661-670.
Sourirajan, A., and M. Lichten, 2008 Polo-like kinase Cdc5 drives exit from pachytene during budding yeast meiosis. Genes Dev. 22: 2627-2632. https://doi.org/10.1101/gad.1711408

Stuckey, S., K. Mukherjee, and F. Storici, 2011 In vivo site-specific mutagenesis and gene collage using the delitto perfetto system in yeast Saccharomyces cerevisiae. Methods Mol. Biol. 745: 173-191. https://doi.org/10.1007/978-1-61779-129-1_11

Subramanian, V. V., and A. Hochwagen, 2014 The meiotic checkpoint network: step-by-step through meiotic prophase. Cold Spring Harb. Perspect. Biol. 6: a016675. https://doi.org/10. 1101/cshperspect.a016675

Subramanian, V. V., A. J. MacQueen, G. Vader, M. Shinohara, A. Sanchez et al., 2016 Chromosome synapsis alleviates Mek1dependent suppression of meiotic DNA repair. PLoS Biol. 14: e1002369. https://doi.org/10.1371/journal.pbio.1002369

Thacker, D., I. Lam, M. Knop, and S. Keeney, 2011 Exploiting sporeautonomous fluorescent protein expression to quantify meiotic chromosome behaviors in Saccharomyces cerevisiae. Genetics 189: 423-439. https://doi.org/10.1534/genetics.111.131326

Tibbles, K. L., S. Sarkar, B. Novak, and P. Arumugam, 2013 CDKdependent nuclear localization of B-cyclin Clb1 promotes FEAR activation during meiosis I in budding yeast. PLoS One 8: e79001. https://doi.org/10.1371/journal.pone.0079001

Tung, K. S., E. J. Hong, and G. S. Roeder, 2000 The pachytene checkpoint prevents accumulation and phosphorylation of the meiosis-specific transcription factor Ndt80. Proc. Natl. Acad. Sci. USA 97: 12187-12192.

Voelkel-Meiman, K., S. S. Moustafa, P. Lefrancois, A. M. Villeneuve, and A. J. MacQueen, 2012 Full-length synaptonemal complex grows continuously during meiotic prophase in budding yeast. PLoS Genet. 8: e1002993. https://doi.org/10.1371/journal. pgen.1002993

Voelkel-Meiman, K., C. Johnston, Y. Thappeta, V. V. Subramanian, A. Hochwagen et al., 2015 Separable crossover-promoting and crossover-constraining aspects of Zip1 activity during budding yeast meiosis. PLoS Genet. 11: e1005335. https://doi.org/ 10.1371/journal.pgen.1005335

Wang, L., Z. Xu, M. B. Khawar, C. Liu, and W. Li, 2017 The histone codes for meiosis. Reproduction 154: R65-R79. https:// doi.org/10.1530/REP-17-0153

Wang, Y., C. Y. Chang, J. F. Wu, and K. S. Tung, 2011 Nuclear localization of the meiosis-specific transcription factor Ndt80 is regulated by the pachytene checkpoint. Mol. Biol. Cell 22: 1878-1886. https://doi.org/10.1091/mbc.E10-12-1011

Weber, C. M., and S. Henikoff, 2014 Histone variants: dynamic punctuation in transcription. Genes Dev. 28: 672-682. https:// doi.org/10.1101/gad.238873.114

White, E. J., C. Cowan, W. Z. Cande, and D. B. Kaback, 2004 In vivo analysis of synaptonemal complex formation during yeast meiosis. Genetics 167: 51-63.

Winter, E., 2012 The Sum1/Ndt80 transcriptional switch and commitment to meiosis in Saccharomyces cerevisiae. Microbiol. Mol. Biol. Rev. 76: 1-15. https://doi.org/10.1128/MMBR.05010-11

Xu, L., B. M. Weiner, and N. Kleckner, 1997 Meiotic cells monitor the status of the interhomolog recombination complex. Genes Dev. 11: 106-118.

Yamada, S., K. Kugou, D. Q. Ding, Y. Fujita, Y. Hiraoka et al., 2018 The histone variant H2A.Z promotes initiation of meiotic recombination in fission yeast. Nucleic Acids Res. 46: 609-620. https://doi.org/10.1093/nar/gkx1110

Communicating editor: J. Engebrecht 